TRANSACTIONS OF THE

AMERICAN MATHEMATICAL SOCIETY

Volume 362, Number 1, January 2010, Pages 261-288

S 0002-9947(09)04814-4

Article electronically published on July 31, 2009

\title{
LOCAL STABILITY OF ERGODIC AVERAGES
}

\author{
JEREMY AVIGAD, PHILIPP GERHARDY, AND HENRY TOWSNER
}

\begin{abstract}
We consider the extent to which one can compute bounds on the rate of convergence of a sequence of ergodic averages. It is not difficult to construct an example of a computable Lebesgue measure preserving transformation of $[0,1]$ and a characteristic function $f=\chi_{A}$ such that the ergodic averages $A_{n} f$ do not converge to a computable element of $L^{2}([0,1])$. In particular, there is no computable bound on the rate of convergence for that sequence. On the other hand, we show that, for any nonexpansive linear operator $T$ on a separable Hilbert space and any element $f$, it is possible to compute a bound on the rate of convergence of $\left\langle A_{n} f\right\rangle$ from $T, f$, and the norm $\left\|f^{*}\right\|$ of the limit. In particular, if $T$ is the Koopman operator arising from a computable ergodic measure preserving transformation of a probability space $\mathcal{X}$ and $f$ is any computable element of $L^{2}(\mathcal{X})$, then there is a computable bound on the rate of convergence of the sequence $\left\langle A_{n} f\right\rangle$.

The mean ergodic theorem is equivalent to the assertion that for every function $K(n)$ and every $\varepsilon>0$, there is an $n$ with the property that the ergodic averages $A_{m} f$ are stable to within $\varepsilon$ on the interval $[n, K(n)]$. Even in situations where the sequence $\left\langle A_{n} f\right\rangle$ does not have a computable limit, one can give explicit bounds on such $n$ in terms of $K$ and $\|f\| / \varepsilon$. This tells us how far one has to search to find an $n$ so that the ergodic averages are "locally stable" on a large interval. We use these bounds to obtain a similarly explicit version of the pointwise ergodic theorem, and we show that our bounds are qualitatively different from ones that can be obtained using upcrossing inequalities due to Bishop and Ivanov.

Finally, we explain how our positive results can be viewed as an application of a body of general proof-theoretic methods falling under the heading of "proof mining."
\end{abstract}

\section{INTRODUCTION}

Let $T$ be a nonexpansive linear operator on a Hilbert space $\mathcal{H}$, that is, a linear operator satisfying $\|T f\| \leq\|f\|$ for all $f \in \mathcal{H}$. For each $n \geq 1$, let $S_{n} f=f+T f+$ $\ldots+T^{n-1} f$ denote the sum of first $n$ iterates of $T$ on $f$, and let $A_{n} f=\frac{1}{n} S_{n} f$ denote their average. The von Neumann mean ergodic theorem asserts that the sequence $\left(A_{n} f\right)$ converges in the Hilbert space norm. The most important instance of the mean ergodic theorem occurs when $\mathcal{H}$ is the space $L^{2}(\mathcal{X})$ of square-integrable functions on a probability space $\mathcal{X}=(X, \mathcal{B}, \mu)$, and $T$ is the Koopman operator $T f=f \circ \tau$ associated to a measure preserving transformation, $\tau$, of that space. In that setting, the Birkhoff pointwise ergodic theorem asserts that the sequence

Received by the editors December 12, 2007.

2000 Mathematics Subject Classification. Primary 37A30, 03F60, 03F03.

Work by the first author was partially supported by NSF grant DMS-0401042.

Work by the second author was partially supported by a postdoctoral grant from the Villum Kann Rasmussen Foundation.

(C)2009 American Mathematical Society 261

Reverts to public domain 28 years from publication 
$\left(A_{n} f\right)$ converges pointwise almost everywhere, and in the $L^{1}$ norm, for any $f$ in $L^{1}(\mathcal{X})$. The transformation $\tau$ is said to be ergodic if there are no nontrivial invariant subsets, in the sense that $\tau^{-1}(A)=A$ implies $\mu(A)=0$ or $\mu(A)=1$. In that case, $\lim _{n \rightarrow \infty} A_{n} f$ is a.e. equal to the constant function $\int f d \mu$, which is to say, almost every sequence of time averages converges to the average over the entire space.

It is known that, in general, the sequence $\left(A_{n} f\right)$ can converge very slowly. For example, Krengel 24] has shown that for any ergodic automorphism of the unit interval under Lebesgue measure and any sequence $\left(a_{n}\right)$ of positive reals converging to 0 , no matter how slowly, there is a subset $A$ of the interval such that, if $\chi_{A}$ denotes the characteristic function of $A$, then

$$
\lim _{n \rightarrow \infty} \frac{1}{a_{n}}\left|\left(A_{n} \chi_{A}\right)(x)-\mu(A)\right|=\infty
$$

almost everywhere, and

$$
\lim _{n \rightarrow \infty} \frac{1}{a_{n}}\left\|A_{n} \chi_{A}-\mu(A)\right\|_{p}=\infty
$$

for every $p \in[1, \infty)$. For related results and references, see [15, Section 0.2] and [25, notes to Section 1.2]. Here, however, we will be concerned with the extent to which a bound on the rate of convergence can be computed from the initial data. That is, given $\mathcal{H}, T$, and $f$ in the statement of the von Neumann ergodic theorem, we can ask whether it is possible to compute, for each rational $\varepsilon>0$, a value $r(\varepsilon)$, such that for every $n$ greater than or equal to $r(\varepsilon)$, we have $\left\|A_{r(\varepsilon)} f-A_{n} f\right\|<\varepsilon$.

Determining whether such an $r$ is computable from the initial data is not the same as determining its rate of growth. For example, if $\left(a_{n}\right)_{n \in \mathbb{N}}$ is any computable sequence of rational numbers that decreases monotonically to 0 , then a rate of convergence can be computed trivially from the sequence: given $\varepsilon$, one need only run through the elements of the sequence and until one of them drops below $\varepsilon$. On the other hand, it is relatively easy to construct a computable sequence $\left(b_{n}\right)$ of rational numbers that converge to 0 , for which there is no computable bound on the rate of convergence. It is also relatively easy to construct a computable, monotone, bounded sequence $\left(c_{n}\right)$ of rationals that does not have a computable limit, which implies that there is no computable bound on the rate of convergence of this sequence, either. These examples are discussed in Section 5

Indeed, in Section [5, we show that there are a computable Lebesgue measure preserving transformation of the unit interval $[0,1]$ and a computable characteristic function $f=\chi_{A}$ such that the limit of the sequence $\left(A_{n} f\right)$ is not a computable element of $L^{2}([0,1])$. For this we rely on standard notions of computability for Hilbert spaces, which we review there. The noncomputability of the limit implies, in particular, that there is no computable bound on the rate of convergence of $\left(A_{n} f\right)$. On the other hand, we show that, for any nonexpansive linear operator $T$ on a separable Hilbert space and any element $f$, one can compute a bound on the rate of convergence of $\left(A_{n} f\right)$ from $T, f$, and the norm $\left\|f^{*}\right\|$ of the limit. In particular, if $T$ is the Koopman operator arising from a computable ergodic measure preserving transformation of a probability space $\mathcal{X}$ and $f$ is any computable element of $L^{2}(\mathcal{X})$, then $\left\|f^{*}\right\|$ is equal to $\left|\int f d \mu\right|$, which is computable; hence there is a computable bound on the rate of convergence.

In situations where the rate of convergence of the ergodic averages is not computable from $T$ and $f$, is there any useful information to be had? The logical 
form of a statement of convergence provides some guidance. The assertion that the sequence $\left(A_{n} f\right)$ converges can be represented as follows:

$$
\forall \varepsilon>0 \exists n \forall m \geq n\left(\left\|A_{m} f-A_{n} f\right\|<\varepsilon\right) .
$$

A bound on the rate of convergence is a function $r(\varepsilon)$ that returns a witness to the existential quantifier for each $\varepsilon>0$. It is the second universal quantifier that leads to noncomputability, since, in general, there is no finite test that can determine whether a particular value of $n$ is large enough. But, classically, the statement of convergence is equivalent to the following:

$$
\forall \varepsilon>0, M: \mathbb{N} \rightarrow \mathbb{N} \exists n\left(M(n) \geq n \rightarrow\left\|A_{M(n)} f-A_{n} f\right\|<\varepsilon\right) .
$$

To see this, note that if, for some $\varepsilon>0$, the existential assertion in (11) is false, then for every $n$ there is an $m \geq n$ such that $\left\|A_{m} f-A_{n} f\right\| \geq \varepsilon$. In that case $\varepsilon$, together with any function $M(n)$ that returns such an $m$ for each $n$, represents a counterexample to (2). Assertion (11) is therefore equivalent to the statement that there is no such counterexample, i.e. assertion (2).

But if the space is explicitly presented and (2) is true, then for each $\varepsilon>0$ and $M$ one can compute a witness to the existential quantifier in (2) by simply trying values of $n$ until one satisfying $\left\|A_{M(n)}-A_{n}\right\|$ is found. Thus, (2) has an inherent computational interpretation. In particular, given any function $K(n)$, suppose we apply (2) to a function $M(n)$ which, for each $n$, returns a value $m$ in the interval $[n, K(n)]$ maximizing $\left\|A_{m} f-A_{n} f\right\|$. In that case, (2) asserts

$$
\forall \varepsilon>0 \exists n \forall m \in[n, K(n)]\left\|A_{m} f-A_{n} f\right\|<\varepsilon .
$$

In other words, if $r(\varepsilon)$ is a function producing a witness to the existential quantifier, then, rather than computing an absolute rate of convergence, $r(\varepsilon)$ provides, for each $\varepsilon>0$, a value $n$ such that the ergodic averages $A_{m} f$ are stable to within $\varepsilon$ on the interval $[n, K(n)]$.

It is now reasonable to ask for an explicit bound on $r(\varepsilon)$, expressed in terms of $K, T, f$, and $\varepsilon$. In Section 2, we obtain bounds on $r(\varepsilon)$ that, in fact, depend only on $K$ and $\rho=\lceil\|f\| / \varepsilon\rceil$. Since the bound on the rate of convergence is clearly monotone with $\rho$, our results show that, for fixed $K$, the bounds are uniform on any bounded region of the Hilbert space and independent of $T$. As special cases, we have the following:

- If $K=n^{O(1)}$, then $r(f, \varepsilon)=2^{2^{O\left(\rho^{2} \log \log \rho\right)}}$.

- If $K=2^{O(n)}$, then $r(f, \varepsilon)=2_{O\left(\rho^{2}\right)}^{1}$, where $2_{n}^{x}$ denotes the $n$th iterate of $y \mapsto 2^{y}$ starting with $x$.

- If $K=O(n)$ and $T$ is an isometry, then $r(f, \varepsilon)=2^{O\left(\rho^{2} \log \rho\right)}$.

Fixing $\rho$ and a parameterized class of functions $K$, one similarly obtains information about the dependence of the bounds on the parameters defining $K$.

In Section 3, we apply the results of Section 2 to the case where $T$ is the Koopman operator corresponding to a measure preserving transformation on a probability space $\mathcal{X}=(X, \mathcal{B}, \mu)$. The pointwise ergodic theorem is equivalent to the assertion that for any $f \in L^{1}(\mathcal{X})$ and for every $\lambda_{1}>0, \lambda_{2}>0$, and $K$, there is an $n$ such that

$$
\mu\left(\left\{x\left|\max _{n \leq m \leq K(n)}\right| A_{m} f(x)-A_{n} f(x) \mid>\lambda_{1}\right\}\right) \leq \lambda_{2} .
$$


When $f$ is in $L^{2}(\mathcal{X})$, we provide explicit bounds on $n$ in terms of $\lambda_{1}, \lambda_{2}, K$, and $\|f\|_{2}$. In this setting, one can obtain similar bounds using alternative methods, namely, from upcrossing inequalities due to Bishop and Ivanov. In Section 4, we show that the bounds extracted using these methods are qualitatively different from the ones obtained using the methods of Sections 2 and 3

Our quantitative versions of the mean and pointwise ergodic theorems are examples of Kreisel's no-counterexample interpretation 22, 23. Our extractions of bounds can be viewed as applications of a body of proof theoretic results that fall under the heading "proof mining" (see, for example, [19, 20, 21]). What makes it difficult to obtain explicit information from the usual proofs of the mean ergodic theorem is their reliance on a nonconstructive principle, namely, the assertion that any bounded increasing sequence of real numbers converges. Qualitative features of our bounds - specifically, the dependence only on $\|f\|, K$, and $\varepsilon$ - are predicted by the general metamathematical results of Gerhardy and Kohlenbach [9. Moreover, methods due to Kohlenbach make it possible to extract useful bounds from proofs that make use of nonconstructive principles such as the one just mentioned. These connections are explained in Section 6 .

In the field of constructive mathematics, one is generally interested in obtaining constructive analogues of nonconstructive mathematical theorems. Other constructive versions of the ergodic theorems, due to Bishop [4, 5, 6, Nuber [27, and Spitters [31, are discussed in Sections 4 and 5. Connections to the field of reverse mathematics are also discussed in Section 5 .

The outline of this paper is as follows. In Sections 2 and 3, we provide our explicit versions of the mean and pointwise ergodic theorems, respectively. In Section 4 , we compare our results to the similar one obtained using upcrossing inequalities. In Section 5 we provide the general computability and noncomputability results alluded to above. In Section 6, we explain the connections to proof mining.

\section{A QUANTITATIVE MEAN ERGODIC THEOREM}

Given any operator $T$ on a Hilbert space and $n \geq 1$, let $S_{n} f=\sum_{i<n} T^{i} f$ and let $A_{n} f=\frac{1}{n} S_{n} f$. The Riesz version of the mean ergodic theorem is as follows.

Theorem 2.1. If $T$ is any nonexpansive linear operator on a Hilbert space and $f$ is any element, then the sequence $\left(A_{n} f\right)$ converges.

We present a proof in a form that will be amenable to extracting a constructive version.

Proof. Let $M=\{f \mid T f=f\}$ be the subspace consisting of fixed points of $T$ and let $N$ be the subspace generated by vectors of the form $u-T u$ (that is, $N$ is the closure of the set of linear combinations of such vectors).

For any $g$ of the form $u-T u$ we have $\left\|A_{n} g\right\|=\frac{1}{n}\left\|u-T^{n} u\right\| \leq 2\|u\| / n$, which converges to 0 . Passing to limits (using the fact that $A_{n}$ satisfies $\left\|A_{n} v\right\| \leq\|v\|$ for any $v$ ), we have that $A_{n} g$ converges to 0 for every $g \in N$.

On the other hand, clearly $A_{n} h=h$ for every $h \in M$. For arbitrary $f$, write $f=g+h$, where $g$ is the projection of $f$ on $N$, and $h=f-g$. It suffices to show 
that $h$ is in $M$. But we have

$$
\begin{aligned}
\|T h-h\|^{2} & =\|T h\|^{2}-2\langle T h, h\rangle+\|h\|^{2} \\
& \leq\|h\|^{2}-2\langle T h, h\rangle+\|h\|^{2} \\
& =2\langle h, h\rangle-2\langle T h, h\rangle \\
& =2\langle h-T h, h\rangle,
\end{aligned}
$$

and the right-hand side is equal to 0 , since $h$ is orthogonal to $N$. So $T h=h$.

The last paragraph of the proof shows that $N^{\perp} \subseteq M$ and, moreover, that $A_{n} f$ converges to $h$. It is also possible to show that $M^{\perp} \subseteq N$, and hence $M=N^{\perp}$, which implies that $h$ is the projection of $f$ on $M$. We will not, however, make use of this additional information below.

As indicated in the Introduction, the mean ergodic theorem is classically equivalent to the following:

Theorem 2.2. Let $T$ and $f$ be as above and let $M: \mathbb{N} \rightarrow \mathbb{N}$ be any function satisfying $M(n) \geq n$ for every $n$. Then for every $\varepsilon>0$ there is an $n \geq 1$ such that $\left\|A_{M(n)} f-A_{n} f\right\| \leq \varepsilon$.

Our goal here is to provide a constructive proof of this theorem that provides explicit quantitative information. We will, in particular, provide bounds on $n$ that depend only on $M$ and $\|f\| / \varepsilon$.

For the rest of this section, we fix a nonexpansive map $T$ and an element $f$ of the Hilbert space. A moment's reflection shows that $A_{n} f$ lies in the cyclic subspace $\mathcal{H}_{f}$ spanned by $\left\{f, T f, T^{2} f, \ldots\right\}$, and so, in the proof of Theorem 2.1, one can replace $N$ by the subspace $N_{f}$ spanned by vectors of the form $T^{i} f-T^{i+1} f$. Let $g$ be the projection of $f$ onto $N_{f}$. Then $g$ is the limit of the sequence $\left(g_{i}\right)_{i \in \mathbb{N}}$, where, for each $i, g_{i}$ is the projection of $f$ onto the finite dimensional subspace spanned by

$$
f-T f, T f-T^{2} f, \ldots, T^{i} f-T^{i+1} f .
$$

The sequence $\left(g_{i}\right)$ can be defined explicitly by

$$
g_{0}=\frac{\langle f, f-T f\rangle}{\|f-T f\|^{2}}(f-T f)
$$

and

$$
g_{i+1}=g_{i}+\frac{\left\langle f-g_{i}, T^{i} f-T^{i+1} f\right\rangle}{\left\|T^{i} f-T^{i+1} f\right\|^{2}}\left(T^{i} f-T^{i+1} f\right) .
$$

For each $i$, we can write $g_{i}=u_{i}-T u_{i}$, where the sequence $\left(u_{i}\right)_{i \in \mathbb{N}}$ is defined by

$$
u_{0}=\frac{\langle f, f-T f\rangle}{\|f-T f\|^{2}} f
$$

and

$$
u_{i+1}=u_{i}+\frac{\left\langle f-g_{i}, T^{i} f-T^{i+1} f\right\rangle}{\left\|T^{i} f-T^{i+1} f\right\|^{2}} T^{i} f .
$$

Note that this representation of $g_{i}$ as an element of the form $u-T u$ is not unique, since if $u$ and $u^{\prime}$ differ by any fixed point of $T, u-T u=u^{\prime}-T u^{\prime}$.

Finally, if we define the sequence $\left(a_{i}\right)_{i \in \mathbb{N}}$ by $a_{i}=\left\|g_{i}\right\|$, then $\left(a_{i}\right)$ is nondecreasing and converges to $\|g\|$. We will see in Section 5 that a bound on the rate of convergence of $\left(a_{i}\right)$ might not be computable from $T$ and $f$. Our strategy here will be to show that, given a fixed "counterexample" function $M$ as in the statement of Theorem 2.2, the fact that the sequence $\left(a_{i}\right)$ is bounded and increasing allows us 
to bound the number of times that $M$ can foil our attempts to provide a witness to the conclusion of the theorem.

First, let us record some easy but useful facts:

Lemma 2.3. $\quad$ 1. For every $n$ and $f,\left\|A_{n} f\right\| \leq\|f\|$.

2. For every $n$ and $u, A_{n}(u-T u)=\left(u-T^{n} u\right) / n$ and $\left\|A_{n}(u-T u)\right\| \leq 2\|u\| / n$.

3. For every $f, g$, and $\varepsilon>0$, if $\|f-g\| \leq \varepsilon$, then $\left\|A_{n} f-A_{n} g\right\| \leq \varepsilon$ for any $n$.

4. For every $f$, if $\langle f, f-T f\rangle \leq \varepsilon$, then $\|T f-f\| \leq \sqrt{2 \varepsilon}$.

Proof. The first two are straightforward calculations, the third follows from the first by the linearity of $A_{n}$, and the fourth follows from calculation (3) in the proof of Theorem 2.1, with $f$ in place of $h$.

Lemma 2.4. For every $f$, if $\|T f-f\| \leq \varepsilon$, then for every $m \geq n \geq 1$ we have $\left\|A_{m} f-A_{n} f\right\| \leq(m-n) \varepsilon / 2$. In particular, if $\|T f-f\| \leq \varepsilon$ and $m \geq 1$, then $\left\|A_{m} f-f\right\| \leq m \varepsilon / 2$.

Proof. Suppose $m \geq n \geq 1$. Then

$$
\begin{aligned}
\left\|A_{m} f-A_{n} f\right\| & =\left\|\frac{1}{m} \sum_{i=0}^{m-1} T^{i} f-\frac{1}{n} \sum_{j=0}^{n-1} T^{j} f\right\| \\
& =\frac{1}{m n}\left\|n \sum_{i=0}^{m-1} T^{i} f-m \sum_{j=0}^{n-1} T^{j} f\right\| \\
& =\frac{1}{m n}\left\|n \sum_{i=n}^{m-1} T^{i} f-(m-n) \sum_{j=0}^{n-1} T^{j} f\right\| .
\end{aligned}
$$

There are now $n \cdot(m-n)$ instances of $T^{i} f$ in the first term and $n \cdot(m-n)$ instances of $T^{j} f$ in the second term. Pairing them off and using the fact that $\left\|T^{i} f-T^{j} f\right\| \leq$ $(i-j) \cdot \varepsilon$ for each such pair, we have

$$
\begin{aligned}
\ldots & \leq \frac{1}{m n}\left(n \sum_{i=n}^{m-1} i-(m-n) \sum_{j=0}^{n-1} j\right) \varepsilon \\
& =\frac{1}{m n}\left(n\left(\frac{m(m-1)}{2}-\frac{n(n-1)}{2}\right)-(m-n)\left(\frac{n(n-1)}{2}\right)\right) \varepsilon \\
& =\frac{1}{m n}\left(n\left(\frac{m(m-1)}{2}\right)-m\left(\frac{n(n-1)}{2}\right)\right) \varepsilon \\
& =(m-n) \varepsilon / 2,
\end{aligned}
$$

as required.

We now turn to the proof of the constructive mean ergodic theorem proper. The first lemma relates changes in $g_{i}$ to changes in $a_{i}$.

Lemma 2.5. Suppose $\left|a_{j}-a_{i}\right| \leq \varepsilon^{2} /(2\|f\|)$. Then $\left\|g_{j}-g_{i}\right\| \leq \varepsilon$.

Proof. Assume, without loss of generality, $j>i$. Since $g_{j}$ is the projection of $f$ onto a bigger subspace, $g_{j}-g_{i}$ is orthogonal to $g_{i}$. Thus, by the Pythagorean theorem, 
we have

$$
\begin{aligned}
\left\|g_{j}-g_{i}\right\|^{2} & =\left\|g_{j}\right\|^{2}-\left\|g_{i}\right\|^{2} \\
& =\left|a_{j}^{2}-a_{i}^{2}\right| \\
& =\left|a_{j}-a_{i}\right| \cdot\left|a_{j}+a_{i}\right| \\
& \leq \frac{\varepsilon^{2}}{2\|f\|} \cdot 2\|f\| \\
& =\varepsilon^{2},
\end{aligned}
$$

as required.

The next lemma introduces a strategy that we will exploit a number of times. Namely, we define an increasing function $F$ such that if, for some $j,\left\|g_{F(j)}-g_{j}\right\|$ is sufficiently small, we have a desired conclusion. We then argue that because the sequence $\left(a_{i}\right)$ is nondecreasing and bounded, sufficiently many iterations of $F$ will necessarily produce such a $j$. (In the next lemma, we use $F(j)=j+1$.)

Lemma 2.6. Let $\varepsilon>0$ and let $d=d(\varepsilon)=\left\lceil 32\|f\|^{4} / \varepsilon^{4}\right\rceil$. Then for every $i$ there $i s$ a $j$ in the interval $[i, i+d)$ such that $\left\|T\left(f-g_{j}\right)-\left(f-g_{j}\right)\right\| \leq \varepsilon$.

Proof. By fact 4 of Lemma 2.3, to obtain the conclusion it suffices to ensure $\langle f-$ $\left.g_{j}, f-g_{j}-T\left(f-g_{j}\right)\right\rangle \leq \varepsilon^{2} / 2$. We have

$$
\begin{aligned}
\left\langle f-g_{j}, f-g_{j}-T\left(f-g_{j}\right)\right\rangle & =\left\langle f-g_{j}, f-T f\right\rangle+\left\langle f-g_{j}, T g_{j}-g_{j}\right\rangle \\
& =\left\langle f-g_{j}, T g_{j}-g_{j}\right\rangle
\end{aligned}
$$

because $g_{j}$ is the projection of $f$ on a space that includes $f-T f$ and $f-g_{j}$ is orthogonal to that space. Recall that $g_{j}$ is a linear combination of vectors of the form $T^{k} f-T^{k+1} f$ for $k \leq j$ and that $g_{j+1}$ is the projection of $f$ onto a space that includes $T g_{j}-g_{j}$. Thus, continuing the calculation, we have

$$
\begin{aligned}
\ldots & =\left\langle f-g_{j+1}, T g_{j}-g_{j}\right\rangle+\left\langle g_{j+1}-g_{j}, T g_{j}-g_{j}\right\rangle \\
& =\left\langle g_{j+1}-g_{j}, g_{j}-T g_{j}\right\rangle \\
& \leq\left\|g_{j+1}-g_{j}\right\| \cdot\left\|T g_{j}-g_{j}\right\| \\
& \leq\left\|g_{j+1}-g_{j}\right\|\left(\left\|T g_{j}\right\|+\left\|g_{j}\right\|\right) \\
& \leq 2\left\|g_{j+1}-g_{j}\right\| \cdot\|f\| .
\end{aligned}
$$

Thus, if $\left\|g_{j}-g_{j+1}\right\| \leq \frac{\varepsilon^{2}}{4\|f\|}$, we have the desired conclusion.

Consider the sequence $a_{i}, a_{i+1}, a_{i+2}, \ldots, a_{i+d-1}$. Since the $a_{j}$ 's are increasing and bounded by $\|f\|$, for some $j \in[i, i+d)$ we have $\left|a_{j+1}-a_{j}\right| \leq \frac{\|f\|}{d} \leq \frac{\varepsilon^{4}}{32\|f\|^{3}}$. By Lemma 2.5, this implies $\left\|g_{j}-g_{j+1}\right\| \leq \frac{\varepsilon^{2}}{4\|f\|}$, as required.

Lemma 2.7. Let $\varepsilon>0$, let $n \geq 1$, and let $d^{\prime}=d^{\prime}(n, \varepsilon)=d(2 \varepsilon / n)=\left\lceil 2 n^{4}\|f\|^{4} / \varepsilon^{4}\right\rceil$. Then for any $i$, there is a $j$ in the interval $\left[i, i+d^{\prime}\right)$ satisfying

$$
\left\|A_{n}\left(f-g_{j}\right)-\left(f-g_{j}\right)\right\| \leq \varepsilon .
$$

Proof. By the previous lemma, there is some $j$ in the interval $\left[i, i+d^{\prime}\right)$ such that $\left\|T\left(f-g_{j}\right)-\left(f-g_{j}\right)\right\| \leq 2 \varepsilon / n$. By Lemma 2.4 this implies $\left\|A_{n}\left(f-g_{j}\right)-\left(f-g_{j}\right)\right\| \leq$ $\varepsilon$. 
Lemma 2.8. Let $\varepsilon>0$, let $m \geq 1$, and let $d^{\prime \prime}=d^{\prime \prime}(m, \varepsilon)=d^{\prime}(m, \varepsilon / 2)=$ $\left\lceil 32 m^{4}\|f\|^{4} / \varepsilon^{4}\right\rceil$. Further suppose $\left\|g_{i}-g_{i+d^{\prime \prime}}\right\| \leq \varepsilon / 4$. Then for any $n \leq m$, $\left\|A_{n}\left(f-g_{i}\right)-\left(f-g_{i}\right)\right\| \leq \varepsilon$.

Proof. By the previous lemma, for any $n \leq m$ there is some $j$ in the interval $\left[i, i+d^{\prime \prime}\right)$ such that $\left\|A_{n}\left(f-g_{j}\right)-\left(f-g_{j}\right)\right\| \leq \varepsilon / 2$. This implies

$$
\begin{aligned}
\left\|A_{n}\left(f-g_{i}\right)-\left(f-g_{i}\right)\right\| \leq & \left\|A_{n}\left(f-g_{i}\right)-A_{n}\left(f-g_{j}\right)\right\| \\
& \quad+\left\|A_{n}\left(f-g_{j}\right)-\left(f-g_{j}\right)\right\|+\left\|\left(f-g_{j}\right)-\left(f-g_{i}\right)\right\| \\
= & \left\|A_{n}\left(g_{j}-g_{i}\right)\right\|+\left\|A_{n}\left(f-g_{j}\right)-\left(f-g_{j}\right)\right\|+\left\|g_{i}-g_{j}\right\| \\
& \leq\left\|A_{n}\left(f-g_{j}\right)-\left(f-g_{j}\right)\right\|+2\left\|g_{j}-g_{i}\right\| \\
& \leq \varepsilon,
\end{aligned}
$$

since $\left\|g_{j}-g_{i}\right\| \leq\left\|g_{i+d^{\prime \prime}}-g_{i}\right\| \leq \varepsilon / 4$.

Lemma 2.9. Let $\varepsilon>0$, and let $m \geq 1$, let $d^{\prime \prime \prime}=d^{\prime \prime \prime}(m, \varepsilon)=d^{\prime \prime}(m, \varepsilon / 2)=$ $\left\lceil 2^{9} m^{4}\|f\|^{4} / \varepsilon^{4}\right\rceil$. Further suppose $\left\|g_{i}-g_{i+d^{\prime \prime \prime}}\right\| \leq \varepsilon / 8$. Then for any $n \leq m$, $\left\|A_{m}\left(f-g_{i}\right)-A_{n}\left(f-g_{i}\right)\right\| \leq \varepsilon$.

Proof. Apply the previous lemma with $\varepsilon / 2$ in place of $\varepsilon$. Then for every $n \leq m$,

$$
\begin{aligned}
&\left\|A_{m}\left(f-g_{i}\right)-A_{n}\left(f-g_{i}\right)\right\| \leq\left\|A_{m}\left(f-g_{i}\right)-\left(f-g_{i}\right)\right\| \\
&+\left\|A_{n}\left(f-g_{i}\right)-\left(f-g_{i}\right)\right\| \\
& \leq \varepsilon / 2+\varepsilon / 2=\varepsilon,
\end{aligned}
$$

as required.

We will not need the following lemma until Section 3, but it is a quick consequence of the preceding result.

Lemma 2.10. Let $\varepsilon>0, m \geq 1, d^{\prime \prime \prime}=d^{\prime \prime \prime}(m, \varepsilon)$ as in Lemma [2.9, $e=\left\lceil\frac{2^{7}\|f\|^{2}}{\varepsilon^{2}}\right\rceil$, and $\hat{d}=\hat{d}(m, \varepsilon)=d^{\prime \prime \prime} \cdot e$. Then for any $i$, there is a $j$ in the interval $[i, i+\hat{d})$ such that for every $n \leq m,\left\|A_{n}\left(f-g_{j}\right)-A_{m}\left(f-g_{j}\right)\right\| \leq \varepsilon$.

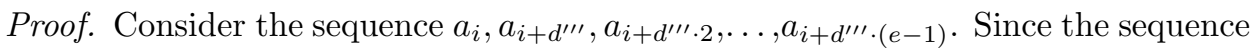
$\left(a_{i}\right)$ is increasing and bounded by $\|f\|$, for some $k<e$ and $j=i+d^{\prime \prime \prime} \cdot k$, we have $\left\|a_{j}-a_{j+d^{\prime \prime \prime}}\right\| \leq \varepsilon^{2} /\left(2^{7}\|f\|\right)$. By Lemma 2.5, this implies $\left\|g_{j}-g_{j+d^{\prime \prime \prime}}\right\| \leq \varepsilon / 8$. Applying the previous lemma with $j$ in place of $i$, we have the desired conclusion.

Let us consider where we stand. Given $\varepsilon>0$ and a function $M$ satisfying $M(n) \geq n$ for every $n$, our goal is to find an $n$ such that $\left\|A_{M(n)} f-A_{n} f\right\| \leq \varepsilon$. Now, for any $n$ and $i$, we have

$$
\begin{aligned}
\left\|A_{M(n)} f-A_{n} f\right\| & =\left\|A_{M(n)}\left(f-g_{i}\right)+A_{M(n)} g_{i}-\left(A_{n}\left(f-g_{i}\right)+A_{n} g_{i}\right)\right\| \\
& \leq\left\|A_{M(n)}\left(f-g_{i}\right)-A_{n}\left(f-g_{i}\right)\right\|+\left\|A_{M(n)} g_{i}\right\|+\left\|A_{n} g_{i}\right\| .
\end{aligned}
$$

Lemma 2.9 tells us how to ensure that the first term on the right-hand side is small: we need only find an $i$ such that $\left\|g_{i+d^{\prime \prime \prime}}-g_{i}\right\|$ is small, for some $d^{\prime \prime \prime}$, depending on $M(n)$, that is sufficiently large. On the other hand, by Lemma 2.3 and $M(n) \geq n$, 
we have $\left\|A_{n} g_{i}\right\| \leq\left\|u_{i}\right\| /(2 n)$ and $\left\|A_{M(n)} g_{i}\right\| \leq\left\|u_{i}\right\| /(2 M(n)) \leq\left\|u_{i}\right\| /(2 n)$. Thus, to guarantee that the remaining two terms are small, it suffices to ensure that $n$ is sufficiently large, in terms of $u_{i}$.

There is some circularity here: our choice of $i$ depends on $M(n)$, and hence $n$, whereas our choice of $n$ depends on $u_{i}$, and hence $i$. The solution is to define sequences $\left(i_{k}\right)_{k \in \mathbb{N}}$ and $\left(n_{k}\right)_{k \in \mathbb{N}}$ recursively, as follows. Set $i_{0}=1$, and, assuming $i_{k}$ has been defined, set

$$
n_{k}=\max \left(\left\lceil\frac{2\left\|u_{i_{k}}\right\|}{\varepsilon}\right\rceil, 1\right)
$$

and

$$
i_{k+1}=i_{k}+d^{\prime \prime \prime}\left(\varepsilon / 2, M\left(n_{k}\right)\right)=i_{k}+\left\lceil\frac{2^{13} M\left(n_{k}\right)^{4}\|f\|^{4}}{\varepsilon^{4}}\right\rceil .
$$

Let $e=\left\lceil 2^{9}\|f\|^{2} / \varepsilon^{2}\right\rceil$, and consider the sequence $a_{i_{0}}, a_{i_{1}}, \ldots, a_{i_{e-1}}$. Once again, since this is increasing and bounded by $\|f\|$, for some $k<e$ we have $\left|a_{i_{k+1}}-a_{i_{k}}\right| \leq$ $\varepsilon^{2} / 2^{9}\|f\|$. Lemma 2.5 implies $\left\|g_{i_{k+1}}-g_{i_{k}}\right\| \leq \varepsilon / 16$. Write $i=i_{k}$ and $n=n_{k}$ so that $i_{k+1}=i+d^{\prime \prime \prime}(M(n), \varepsilon / 2)$. Applying Lemma 2.9, we have

$$
\left\|A_{M(n)}\left(f-g_{i}\right)-A_{n}\left(f-g_{i}\right)\right\| \leq \varepsilon / 2 .
$$

On the other hand, from the definition of $n=n_{k}$, we have

$$
\left\|A_{n} g_{i}\right\| \leq\left\|u_{i}\right\| /(2 n) \leq \varepsilon / 4
$$

and

$$
\left\|A_{M(n)} g_{i}\right\| \leq\left\|u_{i}\right\| /(2 n) \leq \varepsilon / 4
$$

so $\left\|A_{M}(n) f-A_{n} f\right\| \leq \varepsilon$, as required. Notice that the argument also applies for any sequences $\left(i_{k}\right)$ and $\left(n_{k}\right)$ that grow faster than the ones we have defined, that is, satisfy (4) and (5) with "=" replaced by " $\geq$." In sum, we have proved the following:

Lemma 2.11. Given $T, f, \varepsilon$, and $M$, sequences $\left(i_{k}\right)$ and $\left(n_{k}\right)$ as above, and the value $e$ as above, there is an $n$ satisfying $1 \leq n \leq n_{e-1}$ and $\left\|A_{M(n)} f-A_{n} f\right\| \leq \varepsilon$.

This is almost the explicit version of the ergodic theorem that we have promised. The problem is that the bound, $i_{e}$, is expressed in terms of sequence of values $\left\|u_{i}\right\|$ as well as the parameters $M, f$, and $\varepsilon$. The fact that the term $\left\|T^{i} f-T^{i+1} f\right\|$ appears in the denominator of a fraction in the definition of the sequence $\left(u_{i}\right)$ makes it impossible to obtain an upper bound in terms of the other parameters. But we can show that if, for any $i,\left\|T^{i} f-T^{i+1} f\right\|$ is sufficiently small (so $T^{i} f$ is almost a fixed point of $T$ ), we can find alternative bounds on an $n$ satisfying the conclusion of our constructive mean ergodic theorem. Thus we can obtain the desired bounds on $n$ by reasoning by cases: if $T^{i} f-T^{i+1} f$ is sufficiently small for some $i$, we are done; otherwise, we can bound $\left\|u_{i}\right\|$.

The analysis is somewhat simpler in the case where $T$ is an isometry, since then $\left\|T^{i} f-T^{i+1} f\right\|=\|f-T f\|$ for every $i$. Let us deal with that case first. 
Lemma 2.12. If $T$ is an isometry, then for any $m \geq 1$ and $\varepsilon>0$, one of the following holds:

1. $\left\|A_{m} f-f\right\| \leq \varepsilon$, or

2. $\left\|u_{i}\right\| \leq \frac{(i+1) m\|f\|^{2}}{2 \varepsilon}$ for every $i$.

Proof. By the Cauchy-Schwarz inequality we have $\left\|u_{0}\right\| \leq\|f\|^{2} /\|f-T f\|$. By Lemma 2.4 if $\|f-T f\| \leq 2 \varepsilon / m$, then $\left\|A_{m} f-f\right\| \leq \varepsilon$.

Otherwise, $2 \varepsilon / m<\|f-T f\|=\left\|T^{i} f-T^{i+1} f\right\|$ for every $i$. In that case, we have $\left\|u_{0}\right\| \leq \frac{m\|f\|^{2}}{2 \varepsilon}$, and, since $\left\|f-g_{i}\right\| \leq\|f\|$, we obtain

$$
\left\|u_{i+1}\right\| \leq\left\|u_{i}\right\|+\frac{m\|f\|\left\|f-g_{i}\right\|}{2 \varepsilon} \leq\left\|u_{i}\right\|+\frac{m\|f\|^{2}}{2 \varepsilon}
$$

for every $i$. The result follows by induction on $i$.

We can now obtain the desired bounds. If $n=1$ does not satisfy $\| A_{M(n)} f-$ $A_{n} f \| \leq \varepsilon$, we have $n_{k} \leq\left\lceil\frac{\left(i_{k}+1\right) M(1)\|f\|^{2}}{\varepsilon^{2}}\right\rceil$ for each $k$. Otherwise, let $K$ be any nondecreasing function satisfying $K(n) \geq M(n) \geq n$ for every $n$. From the definition of the sequence $\left(i_{k}\right)$, we can extract a function $\widehat{K}(i)$ such that for every $k$, $\widehat{K}^{k}(1) \geq i_{k}$ :

- $\rho=\lceil\|f\| / \varepsilon\rceil$,

- $\widehat{K}(i)=i+2^{13} \rho^{4} K\left((i+1) K(1) \rho^{2}\right)$,

- $e=2^{9} \rho^{2}$.

As long as $f$ is nonzero, we have $\rho \geq 1$, which ensures that $\widehat{K}^{e}(1) \geq n_{e-1}$ and $\widehat{K}^{e}(1) \geq 1$. Thus, we have $\left\|A_{M(n)} f-A_{n} f\right\| \leq \varepsilon$ for some $n \leq \widehat{K}^{e}(1)$.

On the other hand, given a nondecreasing function $K$ to serve as a bound for $M$, the best that a counterexample function $M(n)$ can do is return any $m$ in the interval $[n, K(n)]$ satisfying $\left\|A_{m} f-A_{n} f\right\|>\varepsilon$, if there is one. Thus, we have the following:

Theorem 2.13. Let $T$ be an isometry on a Hilbert space and let $f$ be any nonzero element of that space. Let $K$ be any nondecreasing function satisfying $K(n) \geq n$ for every $n$ and let $\widehat{K}$ be as defined above. Then for every $\varepsilon>0$, there is an $n$ satisfying $1 \leq n \leq \widehat{K}^{e}(1)$, such that for every $m$ in $[n, K(n)],\left\|A_{m} f-A_{n} f\right\| \leq \varepsilon$.

This is our explicit, constructive version of the mean ergodic theorem, for the case where $T$ is an isometry. If $T$ is merely nonexpansive instead of an isometry, the argument is more complicated and requires a more general version of Lemma 2.4.

Lemma 2.14. Assume $T$ is a nonexpansive mapping on a Hilbert space, $f$ is any element, $m \geq n \geq 1$, and $\varepsilon>0$. Then for any $k$, if $n \geq 2 k\|f\| / \varepsilon>k$, then either $\left\|T^{k} f-T^{k+1} f\right\|>\varepsilon /(2 m)$ or $\left\|A_{m} f-A_{n} f\right\| \leq \varepsilon$. 
Proof. We have

$$
\begin{aligned}
\left\|A_{m} f-A_{n} f\right\|= & \frac{1}{m n}\left\|n \sum_{i=0}^{m-1} T^{i} f-m \sum_{j=0}^{n-1} T^{j} f\right\| \\
\leq & \frac{1}{m n}\left\|n \sum_{i=0}^{k-1} T^{i} f-m \sum_{j=0}^{k-1} T^{j} f\right\| \\
& \quad+\frac{1}{m n}\left\|n \sum_{i=k}^{m-1} T^{i} f-m \sum_{j=k}^{n-1} T^{j} f\right\| \\
\leq & \frac{1}{m n}\left\|(n-m) \sum_{j=0}^{k-1} T^{j} f\right\| \\
& \quad+\frac{1}{m n}\left\|n \sum_{i=0}^{m-k-1} T^{i}\left(T^{k} f\right)-m \sum_{j=0}^{n-k-1} T^{j}\left(T^{k} f\right)\right\| .
\end{aligned}
$$

The first term is less than or equal to

$$
\frac{(m-n)}{m n}\left\|\sum_{i=0}^{k-1} T^{j} f\right\| \leq \frac{k\|f\|}{n} \leq \varepsilon / 2 .
$$

Using an argument similar to the one used in the proof of Lemma 2.4, we have

$$
\begin{aligned}
\frac{1}{n m} \| n \sum_{i=0}^{m-k-1} T^{i}\left(T^{k} f\right)-m \sum_{j=0}^{n-k-1} & T^{j}\left(T^{k} f\right) \| \\
& \leq(m-n)\left\|T^{k} f-T^{k+1} f\right\| \leq m\left\|T^{k} f-T^{k+1} f\right\| .
\end{aligned}
$$

If $\left\|T^{k} f-T^{k+1} f\right\| \leq \frac{\varepsilon}{2 m}$, the second term in the last expression is also less than or equal to $\varepsilon / 2$, in which case $\left\|A_{m} f-A_{n} f\right\| \leq \varepsilon$.

We now have an analogue to Lemma 2.12 for the nonexpansive case.

Lemma 2.15. For any $i \geq 0, n \geq 1$, and $\varepsilon>0$, either

1. there is an $n \leq 2 i\left\lceil\frac{\|f\|}{\varepsilon}\right\rceil$ such that $\left\|A_{M(n)} f-A_{n} f\right\| \leq \varepsilon$, or

2. $\left\|u_{i}\right\| \leq \frac{\|f\|^{2}}{2 \varepsilon} \sum_{j=0}^{i} M\left(2 j\left\lceil\frac{\|f\|}{\varepsilon}\right\rceil\right)$.

Proof. Use induction on $i$. At stage $i+1$, if clause 1 doesn't hold, we have $\left\|A_{M(i+1)} f-A_{i+1} f\right\|>\varepsilon$, in which case we can use the inductive hypothesis, the previous lemma, and the definition of $u_{i+1}$ to obtain clause 2 .

The definition of the sequences $\left(i_{k}\right)$ and $\left(n_{k}\right)$ remains valid. What has changed is that we now have a more complex expression for the bounds on $n_{k}$ in the case where case 2 of Lemma 2.15 holds for each $i_{k}$. In other words, we have that for every $k$

$$
n_{k} \leq\left\lceil\frac{\|f\|^{2}}{\varepsilon^{2}} \sum_{l=0}^{i_{k}} M(2 l\lceil\|f\| / \varepsilon\rceil)\right\rceil,
$$


unless there is an $n \leq 2 i_{k}\lceil\|f\| / \varepsilon\rceil$ such that $\left\|A_{M(n)} f-A_{n} f\right\| \leq \varepsilon$. Assuming $K$ is a nondecreasing function satisfying $K(n) \geq M(n)$, we can replace this last bound by $\left\lceil\frac{\|f\|^{2}}{\varepsilon^{2}}\left(i_{k}+1\right) K\left(2 i_{k}\lceil\|f\| / \varepsilon\rceil\right)\right\rceil$. Define

- $\rho=\lceil\|f\| / \varepsilon\rceil$,

- $\bar{K}(i)=i+2^{13} \rho^{4} K\left((i+1) K(2 i \rho) \rho^{2}\right)$,

- $e=2^{9} \rho^{2}$.

Then we have:

Theorem 2.16. Let $T$ be an nonexpansive linear operator on a Hilbert space and let $f$ be any nonzero element of that space. Let $K$ be any nondecreasing function satisfying $K(n) \geq n$ for every $n$ and let $\bar{K}$ be as defined above. Then for every $\varepsilon>0$, there is an $n$ satisfying $1 \leq n \leq \bar{K}^{e}(1)$, such that for every $m$ in $[n, K(n)]$, $\left\|A_{m} f-A_{n} f\right\| \leq \varepsilon$.

Direct calculation yields the following asymptotic bounds.

Theorem 2.17. Let $T$ be any nonexpansive map on a Hilbert space, let $K$ be any nondecreasing function satisfying $K(n) \geq n$ for every $n$, and for every nonzero $f$ and $\varepsilon>0$, let $r_{K}(f, \varepsilon)$ be the least $n \geq 1$ such that $\left\|A_{m} f-A_{n} f\right\| \leq \varepsilon$ for every $m$ in $[n, K(n)]$.

- If $K=n^{O(1)}$, then $r_{K}(f, \varepsilon)=2^{2^{O\left(\rho^{2} \log \log \rho\right)}}$.

- If $K=2^{O(n)}$, then $r_{K}(f, \varepsilon)=2_{O\left(\rho^{2}\right)}^{1}$.

- If $K=O(n)$ and $T$ is an isometry, then $r_{K}(f, \varepsilon)=2^{O\left(\rho^{2} \log \rho\right)}$.

In these expressions, $\rho$ abbreviates $\lceil\|f\| / \varepsilon\rceil$ and $2_{n}^{x}$ denotes the $n$th iterate of $y \mapsto 2^{y}$ starting with $x$.

Alternatively, we can fix $\rho$ and consider the dependence on $K$. Here are two special cases.

Theorem 2.18. Let $T$ be an isometry on a Hilbert space and let $K$ be as above. Fix $\rho=\lceil\|f\| / \varepsilon\rceil$.

- If $K(x)=x+c$, then, as a function of $c, r_{K}(f, \varepsilon)=O(c)$.

- If $K(x)=c x+d$, then, as a function of $c, r_{K}(f, \varepsilon)=c^{O(1)}$.

\section{A quantitative Pointwise ERGodic THEOREM}

Let $\tau$ be a measure preserving transformation on a probability space $(\mathcal{X}, \mathcal{B}, \mu)$ and let $T$ be the Koopman operator on the space $L^{1}(\mathcal{X})$, defined by $T f=f \circ \tau$. The mean ergodic theorem implies that for any $f$ in $L^{2}(\mathcal{X})$, the ergodic averages converge in the $L^{2}$ norm. But since, for any $f$ in $L^{2}(\mathcal{X}),\|f\|_{1} \leq\|f\|_{2}$, this implies convergence in the $L^{1}$ norm also. We also have that $L^{2}(\mathcal{X})$ is dense in $L^{1}(\mathcal{X})$, and if $\left\|f-f^{\prime}\right\|_{1}<\varepsilon$, then $\left\|A_{n} f-A_{n} f^{\prime}\right\|_{1}<\varepsilon$ for every $n$. As a result, we have convergence for every $f$ in $L^{1}(\mathcal{X})$ as well.

Birkhoff's pointwise ergodic theorem makes a stronger assertion:

Theorem 3.1. Let $T$ be the Koopman operator corresponding to a measure preserving transformation $\tau$ on a probability space $\mathcal{X}$, and let $f$ be any element of $L^{1}(\mathcal{X})$. Then $\left(A_{n} f\right)$ converges pointwise, almost everywhere. 
This is equivalent to the following:

Theorem 3.2. Given $T$ and $f$ as above, for every $\lambda_{1}>0$ and $\lambda_{2}>0$ there is an $n$ such that for every $k \geq n$,

$$
\mu\left(\left\{x\left|\max _{n \leq m \leq k}\right| A_{m} f(x)-A_{n} f(x) \mid>\lambda_{1}\right\}\right) \leq \lambda_{2} .
$$

By the logical manipulations described in the Introduction, this, in turn, is equivalent to the following:

Theorem 3.3. Given $T$ and $f$ as above, for every $\lambda_{1}>0, \lambda_{2}>0$, and $K$ there is an $n \geq 1$ satisfying

$$
\mu\left(\left\{x\left|\max _{n \leq m \leq K(n)}\right| A_{n} f(x)-A_{m} f(x) \mid>\lambda_{1}\right\}\right) \leq \lambda_{2} .
$$

Using the maximal ergodic theorem, described below, one can reduce all three versions of the pointwise ergodic theorem to the case where $f \in L^{2}(\mathcal{X})$. In this section, we will provide a constructive proof of Theorem 3.3 for this restricted case, with an explicit bound on $n$, expressed in terms of $K,\|f\|_{2}, \lambda_{1}$, and $\lambda_{2}$.

The maximal ergodic theorem can be stated as follows:

Theorem 3.4. Suppose $n \geq 1$, and let $A=\left\{x \mid \max _{i \leq n} \sum_{j<i} T^{j} f(x)>0\right\}$. Then $\int_{A} f d \mu \geq 0$.

The proof of this is essentially constructive (see [31] and the proofs in [3, 32]). We will make use of the following corollary:

Corollary 3.5. For any $\lambda>0$ and $n \geq 1$,

$$
\mu\left(\left\{x\left|\max _{1 \leq i \leq n}\right| A_{i} f(x) \mid>\lambda\right\}\right) \leq\|f\|_{1} / \lambda .
$$

Proof. We have

$$
\begin{aligned}
\left\{x\left|\max _{1 \leq i \leq n}\right| A_{i} f(x) \mid>\lambda\right\} & \subseteq\left\{x \mid \max _{1 \leq i \leq n} A_{i}(|f|)(x)>\lambda\right\} \\
& =\left\{x \mid \max _{1 \leq i \leq n} A_{i}(|f|-\lambda)(x)>0\right\} \\
& =\left\{x \mid \max _{1 \leq i \leq n} \sum_{j<i} T^{j}(|f|-\lambda)(x)>0\right\} .
\end{aligned}
$$

Call this last set $A$. By Theorem 3.4 we have $\int_{A}(|f|-\lambda) d \mu \geq 0$, and hence

$$
\|f\|_{1}=\int|f| d \mu \geq \int_{A}|f| d \mu \geq \lambda \mu(A),
$$

so $\mu(A) \leq\|f\|_{1} / \lambda$.

Taking limits, Corollary 3.5 implies that for every $\lambda>0$,

$$
\mu\left(\left\{x\left|\sup _{i \geq 1}\right| A_{i} f(x) \mid>\lambda\right\}\right) \leq\|f\|_{1} / \lambda .
$$

We will stick with the formulation above, however, to emphasize the combinatorial character of our arguments.

Most contemporary presentations of the pointwise ergodic theorem proceed to define $f^{*}(x)=\limsup A_{n} f(x)$ and $f_{*}(x)=\liminf A_{n} f(x)$, and then use the maximal ergodic theorem to show that the two are equal almost everywhere. Billingsley 
3], however, presents a proof that makes use of the $L^{2}$ limit of $A_{n} f(x)$, as guaranteed to exist by the mean ergodic theorem, rather than $f^{*}$ and $f_{*}$. We will "mine" this proof for our constructive version.

The idea is as follows. For the moment, let $h$ denote the $L^{2}$ limit of $\left(A_{n} f\right)$, and for each $i$ let $f_{i}=h+g_{i}$, where the sequence $\left(g_{i}\right)$ is as defined in the last section. Then $f=\lim _{i} f_{i}$. For any $m \geq 1, n \geq 1, i \geq 0$, and $x \in \mathcal{X}$, we have

$$
\begin{aligned}
\left|A_{m} f(x)-A_{n} f(x)\right| \leq \mid & A_{m}\left(f-f_{i}\right)(x)|+| A_{m} f_{i}(x)-A_{n} f_{i}(x) \mid \\
& +\left|A_{n}\left(f-f_{i}\right)(x)\right| \\
\leq & \left|A_{m}\left(f-f_{i}\right)(x)\right|+\left|A_{m} h(x)-A_{n} h(x)\right| \\
& \quad+\left|A_{m} g_{i}(x)\right|+\left|A_{n} g_{i}(x)\right|+\left|A_{n}\left(f-f_{i}\right)(x)\right| .
\end{aligned}
$$

Using the maximal ergodic theorem, the first and last terms can be made small outside a small set of exceptions, for all values of $m$ and $n$ simultaneously, by taking $i$ big enough so that $\left\|f-f_{i}\right\|_{2}$ is small. The second term is equal to 0 , since $h$ is a fixed point of $T$. Finally, using the fact that $g_{i}$ is of the form $u-T u$, the third and fourth terms can be made arbitrarily small in the $L^{2}$ norm, and hence small outside a small set of exceptions, by taking $m$ and $n$ sufficiently large.

The problem is that in our constructive version we do not have access to $h$, which is equal to $f-\lim _{i} g_{i}$, nor can we determine how large $i$ has to be to make $\left\|f-f_{i}\right\|_{2}$ sufficiently small. Instead, we replace $h$ by an approximation $f-g_{j}$. Then we get

$$
\begin{aligned}
\left|A_{m} f(x)-A_{n} f(x)\right| \leq \mid & A_{m}\left(g_{j}-g_{i}\right)(x) \mid \\
& +\left|A_{m}\left(f-g_{j}+g_{i}\right)(x)-A_{n}\left(f-g_{j}+g_{i}\right)(x)\right| \\
& +\left|A_{n}\left(g_{j}-g_{i}\right)(x)\right| \\
\leq & A_{m}\left(g_{j}-g_{i}\right)(x)|+| A_{m}\left(f-g_{j}\right)(x)-A_{n}\left(f-g_{j}\right)(x) \mid \\
& +\left|A_{m} g_{i}(x)\right|+\left|A_{n} g_{i}(x)\right|+\left|A_{n}\left(g_{j}-g_{i}\right)(x)\right| .
\end{aligned}
$$

Similar considerations now hold: we can make the first and last terms small outside a small set of exceptions, independent of $m$ and $n$, by ensuring that $\left\|g_{j}-g_{i}\right\|_{2}$ is sufficiently small. We can make the second term small using Lemma 2.10, with an appropriate choice of $j$. Finally, the third and fourth terms are small outside a small set of exceptions when $m$ and $n$ are sufficiently large.

Thus our task is to find a value of $n$ such that

$$
\begin{aligned}
\max _{n \leq m \leq K(n)}\left(\left|A_{m}\left(g_{j}-g_{i}\right)(x)\right|+\right. & \left|A_{m}\left(f-g_{j}\right)(x)-A_{n}\left(f-g_{j}\right)(x)\right| \\
& \left.+\left|A_{m} g_{i}(x)\right|+\left|A_{n} g_{i}(x)\right|+\left|A_{n}\left(g_{l}-g_{i}\right)(x)\right|\right)
\end{aligned}
$$

is less than or equal to $\lambda_{1}$, outside a set of size at most $\lambda_{2}$. We will consider the various components of this sum, in turn.

We will make use of Chebyshev's inequality, which shows that $|f(x)|$ is small, outside a small set of exceptions, when $\|f\|_{2}$ is small:

Lemma 3.6. For any $\lambda \geq 0, \mu(\{x|| f(x) \mid \geq \lambda\}) \leq\|f\|_{2}^{2} / \lambda^{2}$.

Proof. Otherwise, we would have $\|f\|_{2}^{2}=\int|f|^{2} d \mu>\lambda^{2}\left(\|f\|_{2}^{2} / \lambda^{2}\right)=\|f\|_{2}^{2}$. 
The next lemma deals with the first and last terms in (7).

Lemma 3.7. Suppose $\left\|g_{j}-g_{i}\right\|_{2} \leq \lambda_{1} \lambda_{2} / 8$. Then for any $k$ and $n$ satisfying $1 \leq n \leq k$, we have

$$
\mu\left(\left\{x \mid \max _{n \leq m \leq k}\left(\left|A_{m}\left(g_{j}-g_{i}\right)(x)\right|+\left|A_{n}\left(g_{j}-g_{i}\right)(x)\right|\right)>\lambda_{1} / 2\right\}\right) \leq \lambda_{2} / 2 .
$$

Proof. By Corollary 3.5, we have

$$
\mu\left(\left\{x\left|\max _{1 \leq m \leq k}\right| A_{m}\left(g_{j}-g_{i}\right)(x) \mid>\lambda_{1} / 4\right\}\right) \leq\left(\lambda_{1} \lambda_{2} / 8\right) /\left(\lambda_{1} / 4\right)=\lambda_{2} / 2 .
$$

Since $\left|A_{m}\left(g_{j}-g_{i}\right)(x)\right|+\left|A_{n}\left(g_{j}-g_{i}\right)(x)\right|>\lambda_{1} / 2$ implies that either $\left|A_{m}\left(g_{j}-g_{i}\right)(x)\right|>$ $\lambda_{1} / 4$ or $\left|A_{n}\left(g_{j}-g_{i}\right)(x)\right|>\lambda_{1} / 4$, the set in the last displayed formula includes the set in the statement of the lemma.

The next four lemmas concern the third and fourth terms of (7), which are of the form $A_{n} g=A_{n}(u-T u)=\left(u-T^{n} u\right) / n$. To show that we can make these terms small outside a small set by making $n$ sufficiently large, we will need to split $u$ into two components, one of which is bounded and the other of which is small in the $L^{1}$ norm. The following lemma enables us to do this.

Lemma 3.8. For any $u \in L^{2}(\mathcal{X})$ and $L>0$, write $u=u^{\prime}+u^{\prime \prime}$, where

$$
u^{\prime}(x)= \begin{cases}u(x) & \text { if }|u(x)| \leq L, \\ 0 & \text { otherwise }\end{cases}
$$

and $u^{\prime \prime}=u-u^{\prime}$. Then $\left\|u^{\prime}\right\|_{\infty} \leq L$ and $\left\|u^{\prime \prime}\right\|_{1} \leq\|u\|_{2}^{2} / L$.

Proof. The first claim is immediate. For the second, we have

$$
\left\|u^{\prime \prime}\right\|_{1}=\int_{\{x|| u(x) \mid \geq L\}}|u(x)| d \mu \leq \int_{\{x|| u(x) \mid \geq L\}} u^{2}(x) / L d \mu \leq\|u\|_{2}^{2} / L,
$$

as required.

Lemma 3.9. Let $u \in L^{2}(\mathcal{X})$, let $g=u-T u$, and suppose $n \geq \frac{2^{12}\|u\|_{2}^{2}}{\lambda_{1}^{2} \lambda_{2}}$ and $n \geq 1$. Then for any $k \geq n$,

$$
\mu\left(\left\{x \mid \max _{n \leq m \leq k}\left(\left|A_{m} g(x)\right|+\left|A_{n} g(x)\right|\right)>\lambda_{1} / 4\right\}\right) \leq \lambda_{2} / 4 .
$$

Proof. Let $L=2^{7}\|u\|_{2}^{2} / \lambda_{1} \lambda_{2}$ and let $u=u^{\prime}+u^{\prime \prime}$ be the decomposition in Lemma 3.8. Then

$$
\begin{aligned}
\left|A_{m} g_{i}(x)\right|+\left|A_{n} g_{i}(x)\right| \leq\left|A_{m}\left(u^{\prime}-T u^{\prime}\right)(x)\right|+\left|A_{n}\left(u^{\prime}-T u^{\prime}\right)(x)\right| \\
+\left|A_{m}\left(u^{\prime \prime}-T u^{\prime \prime}\right)(x)\right|+\left|A_{n}\left(u^{\prime \prime}-T u^{\prime \prime}\right)(x)\right| .
\end{aligned}
$$

Then we have $\left\|u^{\prime \prime}-T u^{\prime \prime}\right\|_{1} \leq 2\left\|u^{\prime \prime}\right\|_{1} \leq 2\|u\|_{2}^{2} / L$, and so, by Corollary 3.5,

$$
\mu\left(\left\{x\left|\max _{1 \leq n \leq k}\right| A_{n}\left(u^{\prime \prime}-T u^{\prime \prime}\right)(x) \mid>\lambda_{1} / 16\right\}\right) \leq \frac{32\|u\|_{2}^{2}}{L \lambda_{1}}=\lambda_{2} / 4 .
$$

So for $1 \leq n \leq m \leq k$, the sum of the last two terms on the right-hand side of (8) is at most $\lambda_{1} / 8$ outside a set of measure $\lambda_{2} / 4$. On the other hand, for every $x$ and $m \geq n,\left|A_{m}\left(u^{\prime}-T u^{\prime}\right)(x)\right|$ and $\left|A_{n}\left(u^{\prime}-T u^{\prime}\right)(x)\right|$ are bounded by $2\left\|u^{\prime}\right\|_{\infty} / n \leq 2 L / n \leq \lambda_{1} / 16$, since $n \geq 32 L / \lambda_{1}$. 
Taking $k=K(1)$ in the following lemma shows that if $n=1$ does not provide a witness to the conclusion of our constructive pointwise ergodic theorem, we can bound the terms $\left\|u_{i}\right\|_{2}$. This is analogous to Lemma 2.12 .

Lemma 3.10. For any $k \geq 1$, one of the following holds:

1. $\mu\left(\left\{x\left|\max _{1 \leq m \leq k}\right|\left(A_{m} f-f\right)(x) \mid>\lambda_{1}\right\}\right) \leq \lambda_{2}$ or

2. $\left\|u_{i}\right\|_{2} \leq \frac{(i+1)\|f\|_{2}^{2} k^{3 / 2}}{\lambda_{1} \sqrt{\lambda_{2}}}$ for every $i$.

Proof. Suppose, first, $\|T f-f\|_{2} \leq 2 \lambda_{1} \sqrt{\lambda_{2}} / k^{3 / 2}$. Then by Lemma 2.4, for every $m$ satisfying $1 \leq m \leq k$ we have $\left\|A_{m} f-f\right\|_{2} \leq \lambda_{1} \sqrt{\lambda_{2}} / \sqrt{k}$. By Lemma 3.6, this implies

$$
\mu\left(\left\{x||\left(A_{m} f-f\right)(x) \mid>\lambda_{1}\right\}\right) \leq\left(\lambda_{1}^{2} \lambda_{2} / k\right) / \lambda_{1}^{2}=\lambda_{2} / k
$$

for each $m$ in the interval $[1, k]$. So, in that case, clause 1 holds.

Otherwise, we have $\|T f-f\|_{2}>2 \lambda_{1} \sqrt{\lambda_{2}} / k^{3 / 2}$. In that case, clause 2 follows from the definition of the sequence $\left(u_{i}\right)$, as in the proof of Lemma 2.12

Combining the last two lemmas, we have the following.

Lemma 3.11. Either $\mu\left(\left\{x\left|\max _{1 \leq m \leq K(1)}\right|\left(A_{m} f-f\right)(x) \mid>\lambda_{1}\right\}\right) \leq \lambda_{2}$ or, for every $i$, if $n \geq \frac{2^{12} K(1)^{3} i^{2}\|f\|_{2}^{4}}{\lambda_{1}^{4} \lambda_{2}^{2}}$, then for any $k \geq n$ we have

$$
\mu\left(\left\{x \mid \max _{n \leq m \leq k}\left(\left|A_{m} g_{i}(x)\right|+\left|A_{n} g_{i}(x)\right|\right)>\lambda_{1} / 4\right\}\right) \leq \lambda_{2} / 4 .
$$

Finally, we address the second term of (7), using Lemma 2.10

Lemma 3.12. Given $1 \leq n \leq k$, let $e=e\left(k, \lambda_{1}, \lambda_{2}\right)=2^{34} k^{7}\left\lceil\|f\|_{2} /\left(\lambda_{1} \sqrt{\lambda_{2}}\right)\right\rceil^{6}$. Then for any $i$, there is a $j$ in the interval $[i, i+e)$ satisfying

$$
\mu\left(\left\{x\left|\max _{n \leq m \leq k}\right| A_{m}\left(f-g_{j}\right)(x)-A_{n}\left(f-g_{j}\right)(x) \mid>\lambda_{1} / 4\right\}\right) \leq \lambda_{2} / 4 .
$$

Proof. Working backwards, it is enough to ensure that for every $m$ satisfying $n \leq$ $m \leq k$,

$$
\mu\left(\left\{x|| A_{m}\left(f-g_{j}\right)(x)-A_{n}\left(f-g_{j}\right)(x) \mid>\lambda_{1} / 4\right\}\right) \leq \frac{\lambda_{2}}{4 k} .
$$

By Lemma 3.6. it suffices to ensure that

$$
\frac{16\left\|A_{m}\left(f-g_{j}\right)-A_{n}\left(f-g_{j}\right)\right\|_{2}^{2}}{\lambda_{1}^{2}} \leq \frac{\lambda_{2}}{4 k}
$$

for each such $m$, i.e.

$$
\left\|A_{m}\left(f-g_{j}\right)-A_{n}\left(f-g_{j}\right)\right\|_{2} \leq \frac{\lambda_{1} \sqrt{\lambda_{2}}}{8 k} .
$$

Substituting the right-hand side for $\varepsilon$ in Lemma 2.10, this is guaranteed to happen for some $j$ in $[i, i+e)$.

We can finally put all the pieces together. Set $i_{0}=0$. Assuming $i_{k}$ has been defined, let

and

$$
n_{k}=\left\lceil\frac{2^{12} K(1)^{3} i_{k}^{2}\|f\|_{2}^{4}}{\lambda_{1}^{4} \lambda_{2}^{2}}\right\rceil
$$

$$
i_{k+1}=i_{k}+e\left(K\left(n_{k}\right), \lambda_{1}, \lambda_{2}\right)=i_{k}+2^{34} K\left(n_{k}\right)^{7}\left\lceil\|f\|_{2} /\left(\lambda_{1} \sqrt{\lambda_{2}}\right)\right\rceil^{6} .
$$


Finally, define

$$
e=\left\lceil\frac{2^{7}\|f\|_{2}^{2}}{\lambda_{1} \sqrt{\lambda_{2}}}\right\rceil
$$

Then we have:

Lemma 3.13. Let $\lambda_{1}>0$, let $\lambda_{2}>0$, and let $K$ be any function. Given $f$, define the sequence $\left(i_{k}\right)$ and the value $e$ as above. Then there is an $n$ satisfying $1 \leq n \leq n_{e}$ and

$$
\mu\left(\left\{x\left|\max _{n \leq m \leq K(n)}\right| A_{m} f(x)-A_{n} f(x) \mid>\lambda_{1}\right\}\right) \leq \lambda_{2} .
$$

Proof. Suppose $n=1$ does not witness the conclusion. As in the proof of Lemma 2.11, there is some $k<e$ such that $\left|a_{i_{k+1}}-a_{i_{k}}\right| \leq \frac{\lambda_{1}^{2} \lambda_{2}^{2}}{2^{7}\|f\|_{2}}$. Set $n=n_{k}$ and $i=i_{k}$. By Lemma 3.12 there is a $j$ in the interval $\left[i, i_{k+1}\right)$ satisfying

$$
\mu\left(\left\{x\left|\max _{n \leq m \leq K(n)}\right| A_{m}\left(f-g_{j}\right)(x)-A_{n}\left(f-g_{j}\right)(x) \mid>\lambda_{1} / 4\right\}\right) \leq \lambda_{2} / 4 .
$$

Since $i \leq j \leq i_{k+1}$ we have $\left|a_{j}-a_{i}\right| \leq\left|a_{i_{k+1}}-a_{j}\right|$ and so $\left\|g_{i}-g_{j}\right\| \leq \lambda_{1} \lambda_{2} / 8$. By Lemma 3.7, we have

$$
\mu\left(\left\{x \mid \max _{n \leq m \leq K(n)}\left(\left|A_{m}\left(g_{j}-g_{i}\right)(x)\right|+\left|A_{n}\left(g_{j}-g_{i}\right)(x)\right|\right)>\lambda_{1} / 2\right\}\right) \leq \lambda_{2} / 2 .
$$

By Lemma 3.11 we have

$$
\mu\left(\left\{x \mid \max _{n \leq m \leq K(n)}\left(\left|\left(A_{m} g_{i}\right)(x)\right|+\left|\left(A_{n} g_{i}\right)(x)\right|\right)>\lambda_{1} / 4\right\}\right) \leq \lambda_{2} / 4 .
$$

The result follows.

Once again, we can do some final housecleaning. Define:

- $\rho=\left\lceil\|f\| /\left(\lambda_{1} \sqrt{\lambda_{2}}\right)\right\rceil$,

- $\widehat{K}(i)=i+2^{34} \rho^{6} K\left(2^{12} K(1)^{3} i^{2} \rho^{4}\right)$,

- $e=\left\lceil 2^{7}\|f\|_{2}^{2} /\left(\lambda_{1} \sqrt{\lambda_{2}}\right)\right\rceil$.

Theorem 3.14. Let $\tau$ be any measure preserving transformation of a finite measure space $\mathcal{X}$ and let $f$ be any nonzero element of $L^{2}(\mathcal{X})$. Let $\lambda_{1}>0$, let $\lambda_{2}>0$, and let $K$ be any function. Then, with the definitions above, there is an $n$ satisfying $1 \leq n \leq \bar{K}^{e}(1)$ and

$$
\mu\left(\left\{x\left|\max _{n \leq m \leq K(n)}\right| A_{m} f(x)-A_{n} f(x) \mid>\lambda_{1}\right\}\right) \leq \lambda_{2} .
$$

\section{RESUlts FROM UPCROSSING INEQUALITIES}

We are not the first to develop constructive versions of the ergodic theorems. Let $\tau$ be a measure preserving transformation on a finite measure space $\mathcal{X}=(X, \mathcal{B}, \mu)$, and for every real $\alpha<\beta$, let $\omega_{\alpha, \beta}(x)$ denote the number of times the sequence $\left(A_{n} f(x)\right)_{n \in \mathbb{N}}$ upcrosses the interval $[\alpha, \beta]$, that is, proceeds from a value $A_{i} f(x)$ less than $\alpha$ to a value $A_{j} f(x)$ greater than $\beta$. For any $x \in X$, the statement that $\left(A_{n} f(x)\right)_{n \in \mathbb{N}}$ converges is clearly equivalent to the statement that, for every $\alpha<\beta$, $\omega_{\alpha, \beta}(x)$ is finite. Bishop [4, 5, 6] showed that for any $f$ in $L^{1}(\mathcal{X})$, we have

$$
\int_{X} \omega_{\alpha, \beta} d \mu \leq \frac{1}{\beta-\alpha} \int_{X}(f-\alpha)^{+} d \mu .
$$


In particular, let $\Omega_{\alpha, \beta}^{k \uparrow}$ denote the set $\left\{x \mid \omega_{\alpha, \beta}(x) \geq k\right\}$, that is, the set of points for which the sequence makes no less than $k$ upcrossings. Bishop's inequality immediately implies

$$
\mu\left(\Omega_{\alpha, \beta}^{k \uparrow}\right) \leq \frac{1}{k} \frac{1}{\beta-\alpha} \int_{X}(f-\alpha)^{+} d \mu,
$$

from which the ordinary pointwise ergodic theorem follows in a straightforward way. Let the set $\Omega_{[\alpha, \beta]}^{k \downarrow}$ be defined, analogously, to be the set of elements $x$ for which the sequence $\left(A_{n} f(x)\right)_{n \in \mathbb{N}}$ makes no less than $k$ downcrossings from a value above $\beta$ to a value below $\alpha$. Recently, Ivanov [12] has shown that for nonnegative functions $f$, the size of this set decays exponentially with $k$ :

$$
\mu\left(\Omega_{\alpha, \beta}^{k \downarrow}\right) \leq\left(\frac{\alpha}{\beta}\right)^{k}
$$

A similar result was obtained, independently, by Kalikow and Weiss [16]. Bishop's and Ivanov's results and their consequences are explored thoroughly in [15]. There has recently been a surge of interest in such upcrossing inequalities; see, for example, [11, 13, 14].

Upcrossing inequalities can be used in a crude way to obtain bounds on our constructive pointwise ergodic theorem, Theorem 3.3. They can also be used, indirectly, to obtain bounds on our constructive mean ergodic theorem, Theorem 2.2, in the specific case where the operator in question is the Koopman operator corresponding to a measure preserving transformation. Of course, the upcrossing inequalities characterize the overall oscillatory behavior of a sequence, and thus provide more information. On the other hand, our results in Section 2 apply to any nonexpansive mapping on a Hilbert space, and so are more general. There are further differences: because we obtain our pointwise results from our constructive version of the mean ergodic theorem, the $L^{2}$ norm $\|f\|_{2}$ of $f$ plays a central role. In contrast, results obtained using upcrossing techniques are more naturally expressed in terms of $\|f\|_{1}$ and $\|f\|_{\infty}$. In this section, we will see that when the two methods yield analogous results, they provide qualitatively different bounds.

First, let us show how Bishop's inequality leads to a bound on the witness to our constructive pointwise ergodic theorem, Theorem 3.3. when $f$ is in $L^{\infty}(\mathcal{X})$. Note that for any $\beta>\alpha>0$, Bishop's result implies that the number of upcrossings of the interval $[\alpha, \beta]$ satisfies

$$
\int_{X} \omega_{\alpha, \beta} d \mu \leq\|f\|_{\infty} /(\beta-\alpha) .
$$

By symmetry, the same bound holds for downcrossings. Given $\lambda_{1}$, divide the essential range $[-\|f\|,\|f\|]$ of $f$ into $\left\lceil 4\|f\|_{\infty} / \lambda_{1}\right\rceil$ intervals of size $\lambda_{1} / 2$ each. Given $K$ as in Theorem 3.3 . consider the sequence of $e+1$ intervals

$$
[1, K(1)], \quad[K(1), K(K(1))], \ldots, \quad\left[K^{e}(1), K^{e+1}(1)\right] .
$$

For every $i \in[0, e]$ and $j \in\left[1,\left\lceil 4\|f\|_{\infty} / \lambda_{1}\right\rceil\right]$, let $A_{i, j}$ denote the set of $x$ such that $\left(A_{n} f(x)\right)$ upcrosses or downcrosses the $j$ th interval in the essential range of $f$ somewhere in the interval $\left[K^{i}(1), K^{i+1}(1)\right]$. Now, suppose that for each $i \leq e$,

$$
\mu\left(\left\{x\left|\max _{K^{i}(1) \leq m \leq K^{i+1}(1)}\right| A_{m} f(x)-A_{K^{i}(1)} f(x) \mid>\lambda_{1}\right\}\right)>\lambda_{2} .
$$


Since each $x$ in this set either upcrosses or downcrosses one of the $\left\lceil 4\|f\|_{\infty} / \lambda_{1}\right\rceil$ intervals in the range of $f$, we have, for each $i \leq e$,

$$
\sum_{j=1 \ldots\left\lceil 4\|f\|_{\infty} / \lambda_{1}\right\rceil} \mu\left(A_{i, j}\right)>\lambda_{2} .
$$

So

$$
\sum_{i=0 \ldots e, j=1 \ldots\left\lceil 4\|f\|_{\infty} / \lambda_{1}\right\rceil} \mu\left(A_{i, j}\right)>(e+1) \lambda_{2},
$$

which means that for some $j, \sum_{i=0 \ldots e} \mu\left(A_{i, j}\right)>(e+1) \lambda_{1} \lambda_{2} / 4\|f\|_{\infty}$. Let $[\alpha, \beta]$ be the corresponding interval and let $\omega^{\prime}(x)$ be the number of times $\left(A_{n} f(x)\right)$ upcrosses or downcrosses this interval. Then we have

$$
\sum_{i=0 \ldots e} \mu\left(A_{i, j}\right)=\int \sum_{i=0 \ldots e} \chi_{A_{i, j}} d \mu \leq \int \omega^{\prime}(x) d \mu \leq 2\|f\|_{\infty} /(\beta-\alpha)=4\|f\|_{\infty} / \lambda_{1} .
$$

In other words, we have shown that if (9) holds for each $i \leq e$, we have

$$
(e+1) \lambda_{1} \lambda_{2} / 4\|f\|_{\infty} \leq 4\|f\|_{\infty} / \lambda_{1},
$$

which implies $e+1 \leq 16\|f\|_{\infty}^{2} / \lambda_{1}^{2} \lambda_{2}$. Taking the contrapositive of this claim, we have the following analogue of Theorem 3.14

Theorem 4.1. Let $T$ be a Koopman operator corresponding to a measure preserving transformation of a space $\mathcal{X}$ and let $f$ be any element of $L^{\infty}(\mathcal{X})$. Let $\lambda_{1}>0$, let $\lambda_{2}>0$, and let $K$ be any function satisfying $K(n) \geq n$ for every $n$. Let $e=\left\lceil 16\|f\|_{\infty}^{2} / \lambda_{1}^{2} \lambda_{2}\right\rceil$. Then there is an $n$ satisfying $1 \leq n \leq K^{e}(1)$ and

$$
\mu\left(\left\{x\left|\max _{n \leq m \leq K(n)}\right| A_{m} f(x)-A_{n} f(x) \mid>\lambda_{1}\right\}\right) \leq \lambda_{2} .
$$

In other words, we can bound a witness to our constructive pointwise ergodic theorem by $e=\left\lceil 16\|f\|_{\infty}^{2} / \lambda_{1}^{2} \lambda_{2}\right\rceil$ iterations of $K$ on 1 . Compare this to our Theorem 3.14, which requires asymptotically fewer $\left(\left\lceil 2^{7}\|f\|_{2}^{2} / \lambda_{1} \sqrt{\lambda_{2}}\right\rceil\right)$ iterations of a function, $\bar{K}$, which, however, grows faster than $K$.

Ivanov's inequality does not seem to enable us to improve the bound in the previous theorem. But a consequence of Ivanov's inequality, obtained by Kachurovskii, enables us to treat the mean ergodic theorem in a similar way. A sequence of real numbers $a_{n}$ is said to admit $k \varepsilon$-fluctuations if there is a sequence

$$
m_{1}<n_{1} \leq m_{2}<n_{2} \leq \ldots \leq m_{k}<n_{k}
$$

such that for every $i, 1 \leq i \leq k,\left|a_{m_{i}}-a_{n_{i}}\right| \geq \varepsilon$. Let $T$ be the Koopman operator arising from a measure preserving transformation on $\mathcal{X}$. By the mean ergodic theorem, for every $\varepsilon>0$, the number $k_{\varepsilon}$ of $\varepsilon$-fluctuations is finite. Kachurovskii [15. Theorem 29] shows:

Theorem 4.2. Let $f$ be any element of $L^{\infty}(\mathcal{X})$. Then for every $\varepsilon>0$,

$$
k_{\varepsilon} \leq C\left(\frac{\|f\|_{\infty}}{\varepsilon}\right)^{4}\left(1+\ln \left(\frac{\|f\|_{\infty}}{\varepsilon}\right)\right)
$$

for some constant $C$.

Now, given any counterexample function $M$ satisfying $M(n) \geq n$ for every $n$, consider the sequence

$$
A_{1} f, A_{M(1)} f, A_{M(M(1))} f, \ldots, A_{M^{k_{\varepsilon}+1}(1)} f .
$$


At least one step must change by less than $\varepsilon$. Thus, we have the following analogue to our Theorem 2.16 .

Theorem 4.3. Let $T$ be a Koopman operator corresponding to a measure preserving transformation of a space $\mathcal{X}$ and let $f$ be any element of $L^{\infty}(\mathcal{X})$. Let $K$ be any function satisfying $K(n) \geq n$ for every $n$. Let $k(f, \varepsilon)$ be the bound on $k_{\varepsilon}$ given in the preceding theorem. Then for every $\varepsilon>0$, there is an $n, 1 \leq n \leq K^{k(f, \varepsilon)}(1)$, satisfying $\left\|A_{m} f-A_{n} f\right\| \leq \varepsilon$ for every $m \in[n, K(n)]$.

In other words, we can bound a witness to the conclusion of the constructive mean ergodic theorem with $k(f, \varepsilon)$ iterates of $K$. In contrast, Theorem 2.16 required $e(f, \varepsilon)=C\left\lceil\|f\|_{2} / \varepsilon\right\rceil$ iterates of a faster-growing function $\bar{K}$.

\section{Computability of Rates of COnVergence}

Suppose $\left(a_{n}\right)_{n \in \mathbb{N}}$ is any sequence of rational numbers that decreases monotonically to 0 . No matter how slowly the sequence converges, if one is allowed to query the values of the sequence, one can compute a function $r(\varepsilon)$ with the property that for every rational $\varepsilon>0$ and every $m>r(\varepsilon),\left|a_{m}-a_{r(\varepsilon)}\right|<\varepsilon$. The algorithm is simple: on input $\varepsilon$, just search for an $m$ such that $a_{m}<\varepsilon$.

On the other hand, it is not hard to construct a computable sequence $\left(a_{n}\right)_{n \in \mathbb{N}}$ of rational numbers that converges to 0 , with the property that no computable function $r(\varepsilon)$ meets the specification above. This is an easy consequence of the unsolvability of the halting problem. Let $\left(M_{i}\right)_{i>0}$ be an enumeration of Turing machines, and let $j_{i}$ be an enumeration of the natural numbers with the property that every natural number appears infinitely often in the enumeration. For every $i$, let $a_{i}=1 / j_{i}$ if Turing machine $M_{j_{i}}$, when started with input 0 , halts in less than $i$ steps, but not in $i^{\prime}$ steps for any $i^{\prime}<i$ such that $j_{i^{\prime}}=j_{i}$; let $a_{i}=0$ otherwise. Then $\left(a_{j}\right)$ converges to 0 , since once we have recognized all the machines among $M_{1}, \ldots, M_{n}$ that eventually halt, $a_{i}$ remains below $1 / n$. But any value $r(1 / n)$ meeting the specification above tells us how long we have to wait to determine whether $M_{n}$ halts, and so any such such $r$ would enable us to solve the halting problem.

In a similar way, one can construct a computable sequence $\left(a_{n}\right)_{n \in \mathbb{N}}$ of rational numbers that is monotone and bounded, but converges to a noncomputable real number. This, too, implies that no computable function $r(\varepsilon)$ meets the specification above. Such a sequence is known as a Specker sequence, and an example is given in the proof of Theorem 5.1 below. Thus neither monotonicity nor the existence of a computable limit alone is enough to guarantee the effective convergence of a sequence of rationals.

What these examples show is that the question as to whether it is possible to compute a bound on a rate of convergence of a sequence from some initial data is not a question about the speed of the sequence's convergence, but, rather, its predictability. In this section, we show that in general, one cannot compute a bound on the rate of convergence of ergodic averages from the initial data, although one can do so when dealing with an ergodic transformation of a (finite) measure space.

The results in this section presuppose notions of computability for various objects of analysis. There are a number of natural, and equivalent, frameworks for defining 
such notions. For complete details, the reader should consult Pour el and Richards [28] or Weihrauch [33]. To make sense of the results below, however, the following sketchy overview should suffice.

The general strategy is to focus on infinitary objects that can be represented with a countable set of data. For example, a real number can be taken to be represented by a sequence of rational numbers together with a bound on its rate of convergence; the corresponding real number is said to be computable if it has a computable representation. In other words, a computable real number is given by computable functions $a: \mathbb{N} \rightarrow \mathbb{Q}$ and $r: \mathbb{Q} \rightarrow \mathbb{N}$ with the property that for every rational $\varepsilon>0,\left|a_{m}-a_{r(\varepsilon)}\right|<\varepsilon$ for every $m \geq r(\varepsilon)$. A function taking infinitary objects as arguments is said to be computable if the output can be computed by a procedure that queries any legitimate representation of the input. For example, a computable function $f(x)$ from $\mathbb{R}$ to $\mathbb{R}$ is given by an algorithm which, given the ability to request arbitrarily good rational approximations to $x$, produces arbitrarily good rational approximations to $y=f(x)$, in the sense above. In other words, $f$ is given by algorithms that compute functions $a_{y}$ and $r_{y}$ representing $y$, given the ability to query "oracles" $a_{x}$ and $r_{x}$ representing $x$.

Similar considerations apply to separable Hilbert spaces, which are assumed to come with a fixed choice of basis. An element of the space can be represented by a sequence of finite linear combinations of basis elements together with a bound on their rate of convergence in the Hilbert space norm; once again, such an element is said to be computable if it has a computable representation. The inner product and norm are then computable operations on the entire space. A bounded linear operator can be represented by the sequence of values on elements of the basis, and is computable if that sequence is. In general, a computable bounded linear operator need not have a computable norm (see [7, 2]).

Computability with respect to a measure space can be understood in similar ways. A measurable function is represented by a sequence of suitably simple functions that approximate it in the $L^{1}$ norm, together with a rate of convergence. Note that this means that a measurable function is represented only up to a.e. equivalence. One can associate to any measure preserving operator $\tau$ the bounded linear operator $T f=f \circ \tau$, and take $\tau$ to be represented by any representative of the associated $T$.

The following theorem shows that it is not always possible to compute a bound on the rate of convergence of a sequence of ergodic averages from the initial data.

Theorem 5.1. There are a computable measure preserving transformation of $[0,1]$ under Lebesgue measure and a computable characteristic function $f=\chi_{A}$ such that if $f^{*}=\lim _{n} A_{n} f$, then $\left\|f^{*}\right\|_{2}$ is not a computable real number.

In particular, $f^{*}$ is not a computable element of the Hilbert space, and there is no computable bound on the rate of convergence of $\left(A_{n} f\right)$ in either the $L^{2}$ or $L^{1}$ norm. Nor is there a bound on the pointwise rate of convergence of $\left(A_{n} f\right)$, in the sense of Theorem 3.2 .

Proof. It suffices to prove the assertion in the first sentence. The rest of the assertions follow, since if $f^{*}$ were computable, then $\left\|f^{*}\right\|_{2}$ would be computable, and if there were a computable bound on the rate of convergence of $\left(A_{n} f\right)$ in the $L^{2}$ norm, then $f^{*}$ would be a computable element of $L^{2}([0,1])$. Computable bounds on the rate of convergence in either of the other senses mentioned in the remainder 
of the theorem would imply a computable bound on the rate of convergence in the $L^{2}$ norm.

To prove the assertion in the first sentence, we use a variant of constructions described in [2, 29]. First, suppose $f$ is the characteristic function of the interval $[0,1 / 2)$, and $\tau$ is the rotation $\tau x=(x+a) \bmod 1$, where $a$ is either 0 or $1 / 2^{j}$ for some $j \geq 1$. If $a=0$, then $f^{*}=f$ and $\left\|f^{*}\right\|_{2}^{2}=1 / 2$. If $a=1 / 2^{j}$ for any $j \geq 1$, then $f^{*}$ is the constant function equal to $1 / 2$, and $\left\|f^{*}\right\|_{2}^{2}=1 / 4$. Thus knowing $\left\|f^{*}\right\|_{2}$ allows us to determine whether $a=0$.

Our strategy will be to divide $[0,1)$ into intervals $\left[1-2^{i}, 1-2^{i+1}\right)$, and let $\tau$ rotate each interval by a computable real number $a_{i}$ that depends on whether the $i$ th Turing machine halts. With a suitable choice of $f$, the limit $f^{*}$ of the sequence $\left(A_{n} f\right)$ will then encode information as to which Turing machines halt on input 0.

The details are as follows. Let $T(e, x, s)$ be Kleene's $T$ predicate, which asserts that $s$ codes a halting computation sequence of Turing machine $e$ on input $x$. The predicate $T$ is computable, but the set $\{e \mid \exists s T(e, 0, s)\}$ is not. Without loss of generality, we can assume that for any $e$ and $x$ there is at most one $s$ such that $T(e, x, s)$ holds. We will prove the theorem by constructing computable $\tau$ and $f$ such that $\{e \mid \exists s T(e, 0, s)\}$ is computable from $\left\|f^{*}\right\|_{2}$.

Define the computable sequence $\left(a_{i}\right)$ of computable reals by setting

$$
a_{i}= \begin{cases}1 / 2^{i+j+1} & \text { for the unique } j \text { satisfying } T(i, 0, j), \text { if there is one } \\ 0 & \text { otherwise. }\end{cases}
$$

Let $\tau$ be the measure preserving transformation that rotates each interval $\left[1-2^{i}, 1-\right.$ $\left.2^{i+1}\right)$ by $a_{i}$. To see that the sequence $\left(a_{i}\right)$ is computable, remember that we only need to be able to compute approximations to the $a_{i}$ 's uniformly; we can do this by testing $T(i, 0, j)$ up to a sufficiently large value of $j$. To see that $\tau$ is computable, remember that it is sufficient to be able to compute approximations to the value of $T$ applied to any simple function, given rational approximations to the $a_{i}$ 's.

Let $f$ be the characteristic function of the set $\bigcup_{i}\left[1-2^{i}, 1-3 \cdot 2^{i+2}\right)$, so that $f$ is equal to 1 on the left half of each interval $\left[1-2^{i}, 1-2^{i+1}\right)$ and 0 on the right half. Let $f^{*}=\lim _{n} A_{n} f$. Then

$$
\left\|f^{*}\right\|_{2}^{2}=\sum_{\{i \mid \exists j T(i, 0, j)\}} \frac{1}{4} \cdot \frac{1}{2^{i+1}}+\sum_{\{i \mid \neg \exists j T(i, 0, j)\}} \frac{1}{2} \cdot \frac{1}{2^{i+1}}
$$

and

$$
\frac{1}{2}-\left\|f^{*}\right\|_{2}^{2}=\sum_{i \in \mathbb{N}} \frac{1}{2} \cdot \frac{1}{2^{i+1}}-\left\|f^{*}\right\|_{2}^{2}=\sum_{\{i \mid \exists j T(i, 0, j)\}} \frac{1}{2^{i+3}} .
$$

Calling this last expression $r$, it suffices to show that $\{i \mid \exists j T(i, 0, j)\}$ is computable from $r$. But the argument is now standard (see [28, Section 0.2, Corollary 2a] or [30, Theorem III.2.2]). For each $n$, let

$$
r_{n}=\sum_{\{i \mid \exists j \leq n T(i, 0, j)\}} \frac{1}{2^{i+3}} .
$$

Then the sequence $\left(r_{n}\right)$ is computable and increases monotonically to $r$. To determine whether Turing machine $i$ halts on input 0 , it suffices to search for an $n$ and an approximation to $r$ sufficiently good to ensure $\left|r-r_{n}\right|<1 / 2^{i+3}$. Then we only need to check if there is a $j<n$ such that $T(i, 0, j)$ holds; if there isn't, $T(i, 0, j)$ is false for every $j$. 
The proof of Theorem 5.1 relied on the fact that the system we constructed is not ergodic; we used the behavior of the system on each ergodic component to encode the behavior of a Turing machine. The next two theorems and their corollary show that if, on the other hand, the space in question is ergodic, then one always has a computable rate of convergence.

Theorem 5.2. Let $T$ be a nonexpansive linear operator on a separable Hilbert space and let $f$ be an element of that space. Let $f^{*}=\lim _{n} A_{n} f$. Then $f^{*}$, and a bound on the rate of convergence of $\left(A_{n} f\right)$ in the Hilbert space norm, can be computed from $f, T$, and $\left\|f^{*}\right\|$.

Proof. It suffices to show that one can compute a bound on the rate of convergence of $\left(A_{n} f\right)$ from the given data. Assuming $f$ is not already a fixed point of $T$, write $f=f^{*}+g$ and let the sequences $\left(g_{i}\right),\left(u_{i}\right)$, and $\left(a_{i}\right)$ be defined as in Section 2. Then $g=\lim _{i} g_{i}$, and $g_{i}=u_{i}-T u_{i}$ and $a_{i}=\left\|g_{i}\right\|$ for every $i$. Let $a=\lim _{i} a_{i}$. Then $a=\|g\|=\sqrt{\|f\|^{2}-\left\|f^{*}\right\|^{2}}$ can be computed from $f$ and $\left\|f^{*}\right\|_{2}$. For any $m$, $n \geq m$, and $i$, we have

$$
\begin{aligned}
\left\|A_{m} f-A_{n} f\right\| & =\left\|A_{m} g-A_{n} g\right\| \\
& \leq\left\|A_{m} g_{i}-A_{n} g_{i}\right\|+\left\|A_{m}\left(g-g_{i}\right)\right\|+\left\|A_{n}\left(g-g_{i}\right)\right\| \\
& \leq\left\|A_{m} g_{i}\right\|+\left\|A_{n} g_{i}\right\|+2\left\|g-g_{i}\right\| \\
& \leq\left\|A_{m} g_{i}\right\|+\left\|A_{n} g_{i}\right\|+2 \sqrt{2\left(a-a_{i}\right)\|f\|}
\end{aligned}
$$

as in the proof of Lemma 2.5. Given $\varepsilon$, using the given data we can now find an $i$ such that the last term on the right-hand side is less than $\varepsilon / 2$, compute $u_{i}$, and then, using fact 2 Lemma 2.3. determine an $m$ large enough so that for any $n \geq m$, $\left\|A_{m} g_{i}\right\|+\left\|A_{n} g_{i}\right\|<\varepsilon / 2$.

Theorem 5.3. Let $\mathcal{X}=(X, \mathcal{B}, \mu)$ be a separable measure space, let $\tau$ be a measure preserving transformation of $\mathcal{X}$, and let $T$ be the associated Koopman operator. Then for any $f$ in $L^{2}(\mathcal{X})$, bounds on the rate of convergence in the $L^{2}$ norm, in the $L^{1}$ norm, and in the sense of Theorem 3.2 can be computed from $f, T$, and $\left\|f^{*}\right\|_{2}$.

Proof. The previous theorem provides bounds on the rate of convergence in the $L^{2}$ norm, and hence in the $L^{1}$ norm as well.

For convergence in the sense of Theorem 3.2, consider inequality (6), where now $h$ is $f^{*}$ and $f_{i}$ is $g_{i}+f^{*}$ :

$$
\begin{aligned}
\left|A_{m} f(x)-A_{n} f(x)\right| \leq \mid & A_{m}\left(f-\left(g_{i}+f^{*}\right)\right)(x) \mid \\
& +\left|A_{m} g_{i}(x)\right|+\left|A_{n} g_{i}(x)\right|+\left|A_{n}\left(f-\left(g_{i}+f^{*}\right)\right)(x)\right| .
\end{aligned}
$$

The sequence $\left(f-\left(g_{i}+f^{*}\right)\right)_{i \in \mathbb{N}}$ converges to 0 in the $L^{2}$ norm, and, as in the proof of Theorem 5.2, we can compute a bound on the rate of convergence from the given data. Using Corollary [3.5 we can make the first and last terms small outside of a small set of exceptions, independent of $m$ and $n$, by making $i$ sufficiently large. Using Lemma 3.9 we can then determine how large $n$ has to be so that the remaining terms are small outside a small set of exceptions, for all $m>n$.

Corollary 5.4. With $\mathcal{X}=(X, \mathcal{B}, \mu)$ as above, suppose $\tau$ is an ergodic measure preserving transformation. Then for any $f$ in $L^{2}(\mathcal{X})$, bounds on the rate of convergence in the $L^{2}$ norm, in the $L^{1}$ norm, and in the sense of Theorem 3.2 can be computed from $f, T$, and $\mu$. 
For any $f$ in $L^{1}(\mathcal{X})$, bounds on the rate of convergence in the $L^{1}$ norm and in the sense of Theorem 3.2 can be computed from $T, \mu$, and a sequence of $L^{2}(\mathcal{X})$ functions approximating $f$ in the $L^{1}$ norm (together with a rate of convergence).

Proof. If the system is ergodic, $f^{*}$ is a.e. equal to the constant $\int f d \mu$, in which case $\left\|f^{*}\right\|_{2}=\left|\int f d \mu\right|$. Thus $\left\|f^{*}\right\|_{2}$ is computable from $f$ and $\mu$, and we can apply the previous theorem.

Suppose now $f \in L^{1}$ and we are given a sequence $\left(f_{i}\right)$ of $L^{2}$ functions approaching $f$ in the $L^{1}$ norm, together with a rate of convergence. Since

$$
\left\|A_{n} f-A_{n} f_{i}\right\|_{1}=\left\|A_{n}\left(f-f_{i}\right)\right\|_{1} \leq\left\|f-f_{i}\right\|_{1}
$$

for every $n$, we can make $\left\|A_{m} f-A_{n} f\right\|_{1}$ small by first picking $i$ large enough and then ensuring that $\left\|A_{m} f_{i}-A_{n} f_{i}\right\|_{1}$ is small. Similarly, we can make $\left|A_{m} f(x)-A_{n} f(x)\right|$ small outside a small set of exceptions by first choosing $i$ sufficiently large, applying Corollary [3.5. and then using the previous theorem to choose $n$ large enough so that $\left|A_{m} f_{i}(x)-A_{n} f_{i}(x)\right|$ is small outside a small set of exceptions for every $m>n$.

The issues raised here can be considered from a spectral standpoint as well. If $T$ is a unitary transformation of a Hilbert space, then the spectral measure $\sigma_{f}$ associated to $f$ can be described in the following way. For each $k \in \mathbb{Z}$, let $b_{k}=\left\langle T^{k} f, f\right)$ be the $k$ th autocorrelation coefficient of $f$. Let $\mathbb{T}$ be the circle with radius 1 , identified with the interval $[0,2 \pi)$. Let $I$ be the linear operator on the complex Hilbert space $L_{2}^{\mathbb{C}}(\mathbb{T})$ defined with respect to the basis $\left\langle e^{i k \theta}\right)_{k \in \mathbb{Z}}$ by $I\left(e^{i k \theta}\right)=b_{k}$. The sequence $b_{k}$ is a positive definite sequence, and so by Bochner's theorem (see [17, 26]), there is a positive measure $\sigma_{f}$ on $\mathbb{T}$ such that $I(g)=\int g d \sigma_{f}$. It is well known that $\left\|f^{*}\right\|_{2}^{2}=\sigma_{f}(\{0\})$, and Kachurovskii [15, page 670] shows that if $f^{*}=0$, then for every $n$ and $\delta \in(0, \pi)$,

$$
\left\|A_{n} f\right\|_{2} \leq \sqrt{\sigma_{f}(-\delta, \delta)}+\frac{4\|f\|_{2}}{n \sin (\delta / 2)} .
$$

This last expression shows that, in the case where $f^{*}=0$, one can compute a bound on the rate of convergence of $\left(A_{n} f\right)$ from a bound on the rate of convergence of $\sigma_{f}(-\delta, \delta)$ as $\delta$ approaches 0 . The problem is that $I$ is not necessarily a bounded linear transformation, and so $\sigma_{f}$ is not generally computable from $f$. Theorem 5.2 above shows that for any $f$ it is nonetheless possible to compute $f^{*}$ from $\sigma_{f}(\{0\})$, $f$, and $T$.

For any set of natural numbers $X$, let $X^{\prime}$ denote the halting problem relative to $X$. The proof of Theorem 5.1 shows, more generally, the following:

Theorem 5.5. For any set of natural numbers $X$, there are a Lebesgue measure preserving transformation $\tau$ of $[0,1]$, computable from $X$, and a computable element $f$ of $L^{2}([0,1])$, such that $X^{\prime}$ is computable from $\left\|f^{*}\right\|_{2}$.

The results in this section can be adapted to yield information with respect to provability in restricted axiomatic frameworks. Constructive mathematics, for example, aims to use only principles that can be given a direct computational interpretation (see, for example, [5, 7]). There is also a long tradition of developing mathematics in classical theories that are significantly weaker than set theory. In the field of reverse mathematics, this is done with an eye towards calibrating the degree of nonconstructivity of various theorems of mathematics (see [30]); in the 
field of proof mining, this is done with an eye towards mining proofs for additional information (see Section 6, below).

When a theorem of modern mathematics is not constructively valid, one can search for an "equal hypothesis" substitute, i.e. a constructive theorem with the same hypotheses, and with a conclusion that is easily seen to be classically equivalent to the original theorem. Bishop's upcrossing inequalities, as well as the results of Spitters 31, are of this form. The results of Sections 2 and 3 are also of this form, and are provable both constructively and in the weak base theory $R C A_{0}$ of reverse mathematics. One can also look for "equal conclusion" substitutes, by seeking classically equivalent but constructively stronger hypotheses. Theorem 5.2 has this flavor, but it is hard to see how one can turn it into a constructive theorem, because it is not clear how one can refer to $\left\|f^{*}\right\|_{2}$ without presupposing that $\left(A_{n} f\right)$ converges. One can show, constructively and in $R C A_{0}$, that if the projection of $f$ on the subspace $N$ described in the proof of Theorem 2.1 exists, then $\left(A_{n} f\right)$ converges; but the assumption that the projection of $f$ on $M$ exists is not sufficient (see [2, 31] and the corrigendum to the latter). An interesting equal conclusion constructive version of the pointwise ergodic theorem can be found in Nuber [27].

Theorem 5.1 shows that the mean and pointwise ergodic theorems do not have constructive proofs. In fact, in the setting of reverse mathematics, they are equivalent to a set-existence principle known as arithmetic comprehension over $R C A_{0}$. For stronger results, see [2, 29].

\section{Proof-theoretic techniques}

The methods we have used in Sections 2 and 3 belong to a branch of mathematical logic called "proof mining," where the aim is to develop general techniques that allow one to extract additional information from nonconstructive or ineffective mathematical proofs. The program is based on two simple observations: first, ordinary mathematical proofs can typically be represented in formal systems that are much weaker than axiomatic set theory; and, second, proof theory provides general methods of analyzing formal proofs in such theories, with an eye towards locating their constructive content. Traditional research has aimed to show that many classical theories can be reduced to constructive theories, at least in principle, and has developed a variety of techniques for establishing such reductions. These include double-negation translations, cut-elimination, Herbrand's theorem, realizability, and functional interpretations. (The Handbook of Proof Theory [8] provides an overview of the range of methods.) Proof mining involves adapting and specializing these techniques to specific mathematical domains where additional information can fruitfully be sought.

Our constructive versions of the mean and pointwise ergodic theorems are examples of Kreisel's no-counterexample interpretation [22, 23]. Effective proofs of such translated statements can often be obtained using variants of Gödel's functional ("Dialectica") interpretation [10] (see also [1]). Ulrich Kohlenbach has shown that the Dialectica interpretation can be used as an effective tool; see, for example, [19, 21]. For example, our constructive mean ergodic theorem, Theorem 2.16, provides bounds that depend only on $K$ and $\|f\| / \varepsilon$. In fact, the usual proofs of the mean ergodic theorem can be carried out in axiomatic frameworks for which the general metamathematical results of Gerhardy and Kohlenbach 9 guarantee such uniformity. 
While the methods of the paper just cited do show how one can find an explicit expression for the requisite bound, the resulting expression would not yield, a priori, useful bounds. For that, a more refined analysis, due to Kohlenbach [18, can be used. The nonconstructive content of the Riesz proof of the mean ergodic theorem can be traced to the use of the principle of convergence for bounded monotone sequences of real numbers. In formal symbolic terms, the fact that every bounded increasing sequence of real numbers converges can be expressed as follows:

$$
\forall a: \mathbb{N} \rightarrow \mathbb{R}, c \in \mathbb{R}\left(\forall i\left(a_{i} \leq a_{i+1} \leq c\right) \rightarrow \forall \varepsilon>0 \exists n \forall m \geq n\left(\left|a_{m}-a_{n}\right| \leq \varepsilon\right)\right) .
$$

Using a principle known as "arithmetic comprehension," we can conclude that there is a function, $r$, bounding the rate of convergence:

$$
\begin{aligned}
\forall a: \mathbb{N} \rightarrow \mathbb{R}, c \in \mathbb{R}\left(\forall i \left(a_{i} \leq a_{i+1}\right.\right. & \leq c) \rightarrow \\
& \left.\exists r \forall \varepsilon>0 \forall m \geq r(\varepsilon)\left(\left|a_{m}-a_{r(\varepsilon)}\right| \leq \varepsilon\right)\right) .
\end{aligned}
$$

In general, $r$ cannot be computed from the sequence $\left(a_{i}\right)$. On the other hand, the proof of Theorem 5.2 shows that witnesses to the mean ergodic theorem can be computed from a bound $r$ on the rate of convergence, for a sequence $\left(a_{i}\right)$ that is explicitly computed from $T$ and $f$. Moreover, the proof of this fact can be carried out in a weak theory. Kohlenbach's results show that, in such situations, one can compute explicit witnesses to the Dialectica translation to the theorem in question from a weaker version of principle (10):

$$
\begin{aligned}
& \forall a: \mathbb{N} \rightarrow \mathbb{R}, c \in \mathbb{R}\left(\forall i\left(a_{i} \leq a_{i+1} \leq c\right)\right. \rightarrow \\
& \forall \varepsilon>0, M \exists n\left(M(n) \geq n \rightarrow\left(\left|a_{M(n)}-a_{n}\right| \leq \varepsilon\right)\right) .
\end{aligned}
$$

This last principle can be given a clear computational interpretation: given $\varepsilon$ and $M$, one can iteratively compute $0, M(0), M(M(0)), \ldots$ until one finds a value of $n$ such that $\left|a_{M(n)}-a_{n}\right| \leq \varepsilon$. This information can then be used to witness the Dialectica translation of the conclusion, that is, our constructive mean ergodic theorem.

This strategy is clearly in evidence in Section 2. In practice, it is both infeasible and unnecessary to express the initial proof in completely formal terms. Rather, one undertakes a good deal of heuristic manipulation of the original proof, using the translation to determine what form intermediate lemmas should have and how they should be combined. The metamathematical results are therefore used as a guide, providing both guarantees as to what results can be achieved, and the strategies needed to achieve them.

\section{ACKNOWLEDGMENTS}

We are grateful to Ulrich Kohlenbach and James Cummings for comments and corrections.

\section{REFERENCES}

[1] Jeremy Avigad and Solomon Feferman. Gödel's functional ("Dialectica") interpretation. In [8], pages 337-405. MR1640329 (2000b:03204)

[2] Jeremy Avigad and Ksenija Simic. Fundamental notions of analysis in subsystems of secondorder arithmetic. Ann. Pure Appl. Logic, 139:138-184, 2006. MR2206254 (2007f:03098)

[3] Patrick Billingsley. Ergodic Theory and Information. Robert E. Krieger Publishing Co., Huntington, N.Y., 1978. Reprint of the 1965 original. MR.524567 (80b:28017) 
[4] Errett Bishop. An upcrossing inequality with applications. Michigan Math. J., 13:1-13, 1966. MR0194562 (33:2772)

[5] Errett Bishop. Foundations of Constructive Analysis. McGraw-Hill, New York, 1967. MR0221878 (36:4930)

[6] Errett Bishop. A constructive ergodic theorem. J. Math. Mech., 17:631-639, 1967/1968. MR0228655 (37:4235)

[7] Errett Bishop and Douglas Bridges. Constructive Mathematics. Springer, Berlin, 1985. MR804042 (87d:03172)

[8] Samuel R. Buss, editor. The Handbook of Proof Theory. North-Holland, Amsterdam, 1998. MR:1640324 (99d:03051)

[9] Philipp Gerhardy and Ulrich Kohlenbach. General logical metatheorems for functional analysis. Trans. Am. Math. Soc., 360:2615-2660, 2008. MR2373327

[10] Kurt Gödel. Über eine bisher noch nicht benützte Erweiterung des finiten Standpunktes. Dialectica, 12:280-287, 1958. Reproduced with English translation in Feferman et al., eds., Kurt Gödel: Collected Works, volume 2, Oxford University Press, New York, 1990, pages 241-251. MR0102482 (21:1275)

[11] Michael Hochman. Upcrossing inequalities for stationary sequences and applications. arXiv:math/0608311v2.

[12] V. V. Ivanov. Oscillations of averages in the ergodic theorem. Dokl. Akad. Nauk, 347:736-738, 1996. MR1398765 (97d:28021)

[13] Roger L. Jones, Robert Kaufman, Joseph M. Rosenblatt, and Máté Wierdl. Oscillation in ergodic theory. Ergodic Theory Dynam. Systems, 18:889-935, 1998. MR.1645330(2000b:28019)

[14] Roger L. Jones, Joseph M. Rosenblatt, and Máté Wierdl. Counting in ergodic theory. Canad. J. Math., 51:996-1019, 1999. MR1718664(2000i:28021)

[15] A. G. Kachurovski1. Rates of convergence in ergodic theorems. Uspekhi Mat. Nauk, 51:73-124, 1996. Translation in Russian Math. Surveys, 51:653-703, 1996. MR1422228 (2000b:28018)

[16] Steven Kalikow and Benjamin Weiss. Fluctuations of ergodic averages. Illinois J. Math., 43:480-488, 1999. MR 1700603 (2001b:28022)

[17] Yitzhak Katznelson. An Introduction to Harmonic Analysis. Cambridge University Press, Cambridge, third edition, 2004. MR 2039503 (2005d:43001)

[18] Ulrich Kohlenbach. Elimination of Skolem functions for monotone formulas in analysis. Arch. Math. Logic, 37:363-390, 1998. MR1634279 (99m:03119)

[19] Ulrich Kohlenbach. Some logical metatheorems with applications in functional analysis. Trans. Amer. Math. Soc., 357:89-128, 2005. MR2098088 (2005h:03110)

[20] Ulrich Kohlenbach. Applied Proof Theory: Proof Interpretations and their Use in Mathematics. Springer Monographs in Mathematics, Springer-Verlag, Berlin, 2008. MR2445721

[21] Ulrich Kohlenbach. Effective bounds from proofs in abstract functional analysis. To appear in B. Cooper et al., eds., CiE 2005 New Computational Paradigms: Changing Conceptions of What is Computable, Springer, Berlin.

[22] Georg Kreisel. On the interpretation of non-finitist proofs, part I. J. Symbolic Logic, 16:241267, 1951. MR0049135 (14:122k)

[23] Georg Kreisel. Interpretation of analysis by means of constructive functionals of finite type. In Arendt Heyting, editor, Constructivity in Mathematics, pages 101-128. North-Holland, Amsterdam, 1959. MR0106838 (21:5568)

[24] Ulrich Krengel. On the speed of convergence in the ergodic theorem. Monatsh. Math., 86:3-6, 1978/79. MR510630 (80h:28025)

[25] Ulrich Krengel. Ergodic Theorems. Walter de Gruyter \& Co., Berlin, $1985 . \quad$ MR797411 (87i:28001)

[26] M. G. Nadkarni. Spectral Theory of Dynamical Systems. Birkhäuser, Basel, 1998. MR.1719722 (2001d:37001)

[27] J. A. Nuber. A constructive ergodic theorem. Trans. Amer. Math. Soc., 164:115-137, 1972. Erratum: Trans. Amer. Math. Soc., 216:393, 1976. MR0291411 (45:504) MR0382597 $(52: 3479)$

[28] Marian B. Pour-El and J. Ian Richards. Computability in Analysis and Physics. Springer, Berlin, 1989. MR 1005942 (90k:03062)

[29] Ksenija Simic. The pointwise ergodic theorem in subsystems of second-order arithmetic. J. Symbolic Logic, 72:45-66, 2007. MR2298470(2008e:03103) 
[30] Stephen G. Simpson. Subsystems of Second-order Arithmetic. Springer, Berlin, 1999. MR1723993 (2001i:03126)

[31] Bas Spitters. A constructive view on ergodic theorems. J. Symbolic Logic, 71:611-623, 2006. Corrigendum: J. Symbolic Logic 71, 4:1431-1432, 2006. MR2225897(2007b:03105)

[32] Peter Walters. An Introduction to Ergodic Theory. Springer, New York, 2000. MR648108 (84e:28017)

[33] Klaus Weihrauch. Computable Analysis: An Introduction. Springer, Berlin, 2000. MR:1795407 (2002b:03129)

Department of Philosophy, Carnegie Mellon University, Baker Hall 135, PittsBurgh, Pennsylvania 15213

Department of Mathematics, University of Oslo, N-0316 Oslo, Norway

Department of Mathematics, University of California, los Angeles, California 90095- 1555 